\title{
Energy-Efficient Robust Control for Direct Drive and Energy Recuperation Hydraulic Servo System
}

\author{
Weiping Wang $\mathbb{D}^{1,2}$ and Jiyun Zhao ${ }^{2}$ \\ ${ }^{1}$ School of Mechatronic Engineering, Jiangsu Normal University, Xuzhou 221116, China \\ ${ }^{2}$ School of Mechatronic Engineering, China University of Mining and Technology, Xuzhou 221116, China \\ Correspondence should be addressed to Weiping Wang; kuangdaxiao5@cumt.edu.cn
}

Received 7 October 2019; Revised 20 December 2019; Accepted 31 December 2019; Published 14 February 2020

Academic Editor: Mohammad Hassan Khooban

Copyright ( 92020 Weiping Wang and Jiyun Zhao. This is an open access article distributed under the Creative Commons Attribution License, which permits unrestricted use, distribution, and reproduction in any medium, provided the original work is properly cited.

During the vertical cyclic actuation process of heavy materials handling, the gravitational potential energy will be converted to heat in the form of throttle in the traditional hydraulic system. Therefore, large energy dissipation is inevitable is this process. In order to achieve energy recuperation, as well as precise trajectory tracking, a direct drive and energy recuperation system is developed. To be specific, the function of direct drive and energy recovery is realized via the three-chamber actuator and a hydraulic accumulator. Moreover, the optimized parameters are obtained by the simulated annealing algorithm. To further reduce the energy consumption, the variable supply pressure control circuit is introduced into the system. Furthermore, the prescribed tracking performance is guaranteed by the proposed robust controller. To compensate for the uncertainties, both in the variable supply pressure control circuit and the robust controller, the RBF neural network is employed to approximate the unknown function. The presented approach theoretically possesses the ability to minimize the energy consumption while maintaining satisfied tracking accuracy. The results demonstrate that the proposed approach can save nearly 90 percent of the energy, and the maximum tracking error is $2 \mathrm{~mm}$.

\section{Introduction}

Hydraulic actuation has numerous applications in mobile machinery and industrial engineering such as steering system, heavy-duty robot, press, and excavator [1-4]. Different from other driving form, the fluid power system usually suffers from poor energy efficiency. Therefore, reducing energy consumption and pollution emissions is becoming an imperative problem for the hydraulically actuated system, especially under the background of energy exhaustion [5-11].

As an important part of the fluid power system, the hydraulic servo system has attracted a wide spread of attention in many years $[12,13]$. However, the existing literatures regarding the hydraulic servo system usually concentrated on the improvement of tracking performance due to the difficulties result from the highly nonlinear nature of the plant. Actually, the energy consumption is also a big issue that needs to be dealt with [14-17]. To achieve the two targets simultaneously, both the control law and the hardware configuration should be taken into account.
Generally, the energy consumption of the hydraulic servo system mainly results from the overflow loss and the throttle loss. For the sake of decreasing the overflow loss and throttle loss simultaneously, the variable-displacement-pump-based system has been extensively studied, and this method can significantly improve the system efficiency [18]. A load sensing variable pump was utilized to achieve the energy saving effect during the tracking task [19]. Moreover, the electrohydraulic load-sensing (EHLS), which comprises the frequency converter and fixed displacement pump, was also employed to reduce the energy consumption in [20]. Although the aforementioned methods can achieve satisfied energy-saving effect, the system bandwidth is much lower than the valvecontrolled system due to high inertias [21]. Since the system bandwidth is of great importance for hydraulic servo system, the pump-controlled system is inferior to the valve-controlled system in this regard. Moreover, the high installation cost of this kind of system is also worthy to be considered.

As an improved form of EHLS, the variable supply pressure control circuit (VSPC) has been widely investigated in recent years. Typically, the system comprises of a constant 
displacement pump and the supply pressure is regulated by the proportional relief valve. In [22], a LQR controller-based VSPC is presented to improve the system efficiency as well as the tracking performance. In [23], the control law of VSPC can also be deduced by assuming the desired spool position. In addition, a load-prediction-based VSPC controller was developed for a hydraulic actuated robot so as to enhance the energy saving capability of the system [24]. In our previous studies, two kinds of VSPC, which combined with the disturbance observer and state observer, were also proposed $[25,26]$.

Despite the fact that the aforementioned VSPC can greatly diminish the energy consumption, it does not work on gravitational potential energy recovery [27]. Numerous hydraulic equipments such as vibration test bench, energy regenerative suspension, fast forging machine, and hydraulic press have the characteristic of vertical reciprocating motion. If the gravitational potential energy is not properly handled, there will be large energy consumption [28-31]. In order to address this problem, the independent metering technique is introduced [32, 33]. Specifically, an energysaving adaptive robust control of a hydraulic manipulator using five cartridge valves and a hydraulic accumulator is proposed in [34, 35]. Moreover, a direct driven hydraulic pump-controlled system based on the load compensating circuit is presented in $[36,37]$.

As reviewed above, it is necessary to minimize the energy consumption under the premise of ensuring the tracking performance. For this purpose, both the hardware configuration and the control strategy should be taken into consideration. Therefore, a direct drive and energy recuperation hydraulic servo system as well as its corresponding robust controller is presented in this study so as to achieve the multiobjective task.

The main contributions of this study can be summarized as follows:

(1) The energy recovery hardware, which consists of a three-chamber actuator and a hydraulic accumulator, is presented. It is able to recycle and reuse the gravitational potential energy directly. Moreover, the parameter configuration is optimized by the simulated annealing algorithm.

(2) The proposed control strategy is able to reduce the throttle loss according to the VSPC, and the uncertainties in the control law is compensated by the RBF neural network.

(3) The robust control strategy based on the compensation effect of the RBF neural network can guarantee a prescribed transient performance and final tracking precision.

The rest of the paper is organized as follows. Section 2 introduces the proposed hardware configuration and the control objective. The mathematical model is developed in Section 3. The simulated annealing algorithm and VSPC approach is introduced in Sections 4 and 5, respectively. The robust controller as well as the RBF neural network is developed in Section 6. Section 7 demonstrates the results of case studies. The brief conclusions are summarized in Section 8 .

\section{Problem Formulation}

The simplified schematic of the proposed system is depicted in the left side of Figure 1(a), and the traditional twochamber hydraulic actuator is presented in Figure 1(b). The proposed actuator possesses three working chambers, which are piston chamber, rod chamber, and storage chamber. The storage chamber is connected to a hydraulic accumulator so as to directly achieve energy recuperation and release. To be specific, when the actuator is retracted, the potential energy of the mass load will be converted to hydraulic energy of the accumulator via the storage chamber. Moreover, the accumulator will release the stored energy to assist the system during the lifting stage. Although there exists many literatures regarding hydraulic accumulator-based energy recovery, the energy conversion or transition link such as the hydraulic transformer or inverter is required. Therefore, part of the energy will be wasted in these kinds of systems. The first two chambers are connected with the variable supply pressure control circuit (VSPC), which mainly consists of a proportional directional valve (PDV), a proportional relief valve (PRV), and a fixed displacement pump. The VSPC is able to regulate the supply pressure according to the demand. Therefore, the pump only needs to provide a low pressure in the entire working process.

The multiobjective control task can be concluded as follows: given the desired tracking trajectory, the task is to synthesize a control input for PDV such that the actuator can precisely track the desired trajectory. In the meantime, the controller generates a control signal for PRV in order to reduce the energy consumption.

\section{System Modelling}

System modeling is the basis of parameter optimization and control strategy design. Therefore, the complete mathematical model of the system is given below.

According to Boyle's theorem, the pressure in the hydraulic accumulator can be calculated as [38]

$$
p_{0} V_{0}^{n}=p_{a 1} V_{a 1}^{n}=p_{a 2} V_{a 2}^{n}=p_{a} V_{a}^{n}
$$

where $n$ is the gas exponent, $V_{0}$ is the volume of the hydraulic accumulator, $p_{0}$ is the precharge pressure of the hydraulic accumulator, $p_{a 1}$ and $p_{a 2}$ are the minimum working pressure and the maximum working pressure of the hydraulic accumulator, respectively, $V_{a 1}$ and $V_{a 2}$ are the corresponding gas volume, $p_{a}$ and $V_{a}$ are the pressure and gas volume of the hydraulic accumulator at any given moment, respectively.

The model of the hydraulic accumulator can be deduced from equation (1), which is

$$
\dot{p}_{a}=\frac{p_{0} n}{V_{0}} Q_{a}=-\frac{p_{0} n}{V_{0}} A_{a} \dot{x}_{p}
$$

where $Q_{a}$ is the flow of the hydraulic accumulator, $x_{p}$ is the displacement of the cylinder $\operatorname{rod}$, and $A_{a}$ is the effective area of the storage chamber.

Based on equation (1), the effective working volume of the hydraulic accumulator can be derived as follows [36]: 


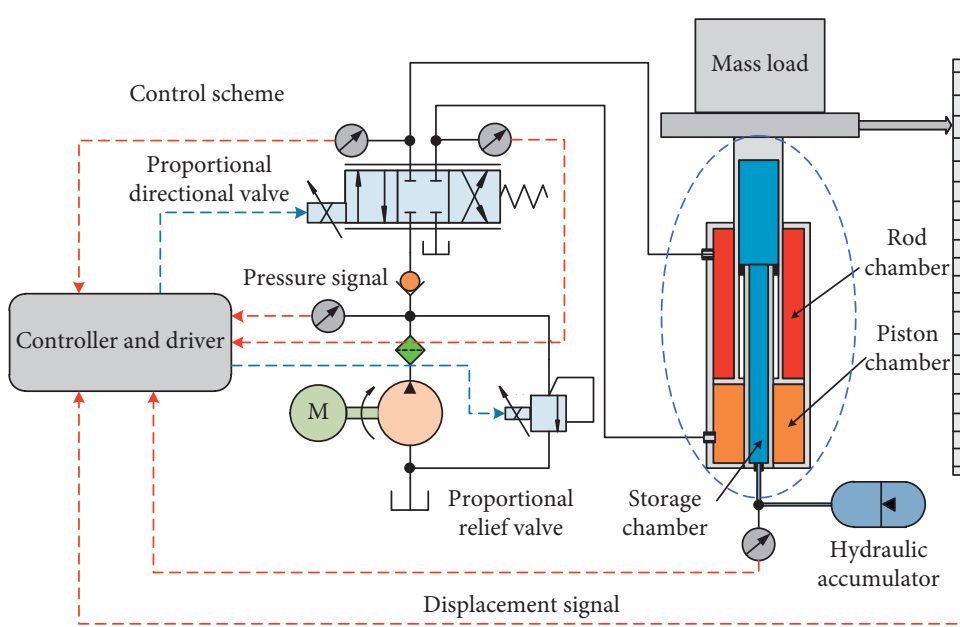

(a)

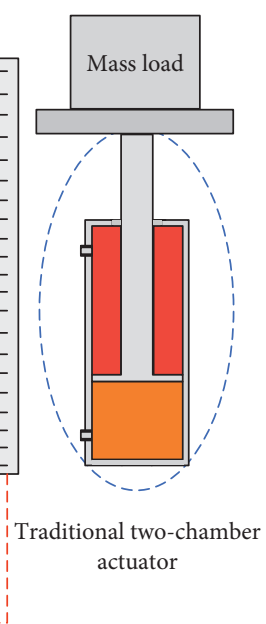

(b)

FIGURE 1: Simplified schematic of the proposed direct drive and energy recuperation hydraulic servo system (a) and the traditional twochamber hydraulic actuator (b).

$$
V_{w}=V_{a 1}-V_{a 2}=A_{a} L=\left[\left(\frac{p_{0}}{p_{a 1}}\right)^{1 / n}-\left(\frac{p_{0}}{p_{a 2}}\right)^{1 / n}\right] V_{0},
$$

where $V_{w}$ is the effective working volume of the hydraulic accumulator and $L$ is the stroke of the actuator.

The force balance equation of the system is expressed as

$$
\begin{aligned}
m \ddot{x}_{p}= & p_{1} A_{1}-p_{2} A_{2}+p_{a} A_{a}-G-B_{c} \dot{x}_{p}-k x_{p} \\
& +F_{d}+\Delta_{1 n}+\Delta_{1 s},
\end{aligned}
$$

where $m$ is the mass of the load, $p_{1}$ and $p_{2}$ are the pressures of piston chamber and rod chamber, respectively, $A_{1}$ and $A_{2}$ are the corresponding effective areas, $B_{c}$ is the coefficient of viscous damping, $k$ is the environment stiffness, $G$ is the equivalent gravity of the mass load, $F_{d}$ is the external disturbance, and $\left(\Delta_{1 n}+\Delta_{1 s}\right)$ represents the lumped uncertainties including parametric uncertainties and uncertain nonlinearities, in which $\Delta_{1 n}$ denotes its nominal value.

Since the natural frequency of the servovalve used here is much higher that of a typical EHSS, the valve dynamic is often neglected without a significant reduction in the control performance [35]. Therefore, the flow equations of the hydraulic valve can be written as

$$
\begin{aligned}
& \left\{\begin{array}{l}
Q_{1}=k_{q} k_{x} \underbrace{\left[s_{g}(u) \sqrt{\frac{2}{\rho}\left(p_{s}-p_{1}\right)}+s_{g}(-u) \sqrt{\frac{2}{\rho}\left(p_{1}-p_{t}\right)}\right]}_{g_{1}} u, \\
Q_{2}=k_{q} k_{x} \underbrace{\left[s_{g}(u) \sqrt{\frac{2}{\rho}\left(p_{2}-p_{t}\right)}+s_{g}(-u) \sqrt{\frac{2}{\rho}\left(p_{s}-p_{2}\right)}\right]}_{g_{2}} u,
\end{array}\right. \\
& s_{g}(\cdot)= \begin{cases}1, & \text { if } \cdot \geq 0, \\
0, & \text { if } \cdot<0,\end{cases}
\end{aligned}
$$

where $k_{q}$ is the flow gain coefficient of the servovalve, $k_{x}$ is a positive constant, $p_{s}$ is the supply pressure of the pump, $p_{t}$ is the tank pressure, $\rho$ is the oil density, $Q_{1}$ is the supply flow rate to the forward chamber, $Q_{2}$ is the return flow rate from the return chamber, and $u$ is the input signal of PDV.

The pressure dynamics of the piston chamber and rod chamber can be written as [39]

$$
\left\{\begin{array}{l}
\dot{p}_{1}=c_{1}\left(Q_{1}-A_{1} \dot{x}_{p}\right)+\Delta_{2}, \\
\dot{p}_{2}=c_{2}\left(-Q_{2}+A_{2} \dot{x}_{p}\right)+\Delta_{3},
\end{array}\right.
$$

where $c_{1}=\beta_{e} / V_{1}, c_{2}=\beta_{e} / V_{2}, V_{1}$ and $V_{2}$ are the control volumes of the two chambers, respectively, $\beta_{e}$ is the effective bulk modulus of the system, and $\Delta_{2}$ and $\Delta_{3}$ denote the lumped uncertainties including external disturbance and terms like the neglected items related to leakage.

In order to make the system fall into the strict feedback form, the state variables are defined as follows:

$$
\left[x_{1}, x_{2}, x_{3}\right]^{T}=\left[x_{p}, \dot{x}_{p}, p_{1}-a_{1} p_{2}+a_{2} p_{a}\right]^{T},
$$

where $a_{1}=A_{2} / A_{1}$ and $a_{2}=A_{a} / A_{1}$.

Therefore, the entire system can be expressed in a state space form as follows:

$$
\begin{aligned}
& \left\{\begin{array}{l}
\dot{x}_{1}=x_{2}, \\
\dot{x}_{2}=\frac{A_{1}}{m} x_{3}-\frac{B_{c}}{m} x_{2}-\frac{k}{m} x_{1}-\frac{G}{m}+\underbrace{\frac{F_{d}+\Delta_{1 n}}{m}}_{\varphi}+\underbrace{\frac{\Delta_{1 s}}{m}}_{d_{1}}, \\
\dot{x}_{3}=f_{1} u-f_{2} x_{2}+\underbrace{\Delta_{2}-a_{1} \Delta_{3}}_{d_{2}}, \\
y=x_{1},
\end{array}\right. \\
& \left\{\begin{array}{l}
f_{1}=c_{1} k_{q} k_{x} g_{1}+a_{1} c_{2} k_{q} k_{x} g_{2}, \\
f_{2}=A_{1} c_{1}+a_{1} c_{2} A_{2}+\frac{a_{2} p_{0} n A_{a}}{V_{0}},
\end{array}\right.
\end{aligned}
$$


where $f_{1} \sim f_{2}$ are shown in equation (9), and $\varphi$ are unknown functions.

Assumption 1. The extent of $d_{1}, d_{2}$, and $\varphi$ is known, i.e., $\left|d_{i}\right| \leq D_{i} i=1,2$ and $\varphi_{\min } \leq \varphi \leq \varphi_{\max }$.

\section{Parameter Optimization Based on Simulated Annealing Algorithm}

According to the mathematical model deduced in the previous section, it can be inferred that the parameters of the hydraulic accumulator and actuator may greatly affect the energy-saving performance. Therefore, it is indispensable to optimize the parameters.

Ideally, the force provided by the hydraulic accumulator can exactly balance the gravity of the mass load. Accordingly, the supply pressure can be very small either in the retracted stage or in the lifting stage. However, it is almost impossible since the pressure of the hydraulic accumulator varies consistently and it cannot precisely match the load.

If the total driving force provided by the system exactly balances the load, the actuator will moves at a constant speed. This means the discrepancy between the force provided by the hydraulic accumulator and the load gravity are compensated by the VSPC. Since this part of energy is only utilized for balance rather than drive the actuator to track the given trajectory, and it is regarded as energy dissipation. Therefore, the optimization objective is to reduce the excess energy supply as much as possible.

Generally, there are three design schemes for the hydraulic accumulator.

Case 1. the force provided by the hydraulic accumulator is always higher than the load gravity (blue line in Figure 2).

Case 2. there is a crossover between the force provided by the hydraulic accumulator and the load (red line in Figure 2).

Case 3. the force provided by the hydraulic accumulator is always lower than the load gravity (purple line in Figure 2).

Take case 1 as an example; in the retracted stage of case 1 , the VSPC should provide a high pressure for the rod chamber so as to balance the force provided by the hydraulic accumulator. On the contrary, in the lifting stage, the system barely needs to provide energy for the piston chamber. Instead, the VSPC may even need to limit the velocity of the actuator by throttle. It is because the energy stored in the hydraulic accumulator is sufficient to drive the mass load on its own. It can be seen from Figure 2 that the wasted energy in case 1 is the enclosed area between force of the hydraulic accumulator and the load force. Other cases can be analyzed by analogy. It can be inferred from comparison that case 2 is the best scheme, and the green area is the excess energy consumption.

In order to obtain the minimum energy consumption, the parameters should be optimized. Actually, space limitation and physical factor should be taken into consideration

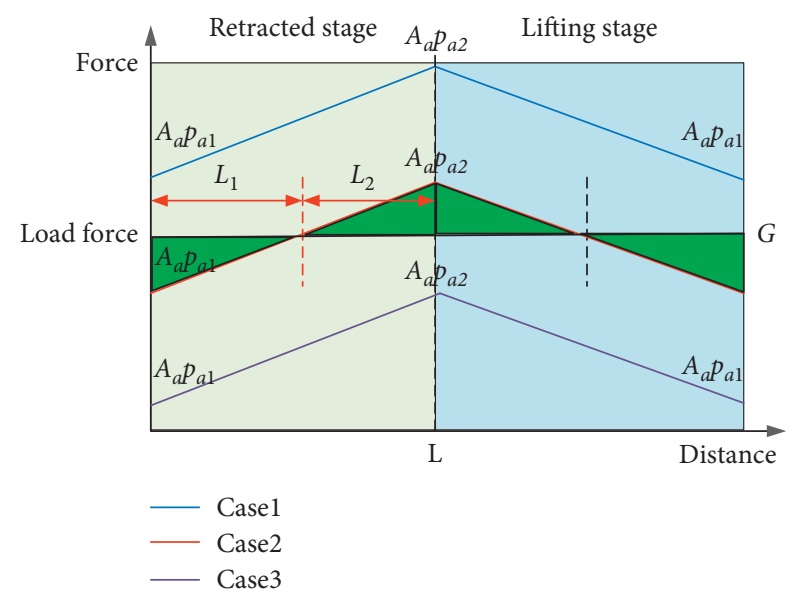

FIgURE 2: Comparison of different design schemes of the hydraulic accumulator.

when selecting a hydraulic accumulator. Therefore, the constraint conditions of the parameters and the objective function are given in the following equation:

$$
\left\{\begin{array}{l}
V_{0 \min } \leq V_{0} \leq V_{0 \max }, \\
A_{a \min } \leq A_{a} \leq A_{a \max } \stackrel{\min }{\longrightarrow} f=\left(G-p_{a 1} A_{a}\right) L_{1}+\left(p_{a 2} A_{a}-G\right) L_{2}, \\
p_{0 \min } \leq p_{0} \leq p_{0 \max }, \\
p_{0}=\alpha p_{a 1},
\end{array}\right.
$$

where $V_{0 \text { max }}, A_{a \max }$, and $p_{0 \max }$ are the upper limit values and $V_{0 \text { min }}, A_{a \min }$, and $p_{0 \text { min }}$ are the lower limit values.

The objective function is derived as follows.

The maximum working pressure $p_{a 2}$ can be deduced from equation (3), which is

$$
p_{a 2}=\frac{p_{0}}{\left[\left(p_{0} / p_{a 1}\right)^{1 / n}-\left(A_{a} L / V_{0}\right)\right]^{n}} .
$$

It can be seen from equation (2) that if the actuator moves at a constant speed, the pressure dynamic of the hydraulic accumulator can be regarded as a constant. Therefore, $L_{1}$ and $L_{2}$ can be calculated as

$$
\begin{aligned}
& L_{2}=\frac{L}{\left(G-p_{a 1} A_{a} / p_{a 2} A_{a}-G\right)+1}, \\
& L_{1}=L-L_{2} .
\end{aligned}
$$

Based on the aforementioned analysis, the objective function in equation (10) can be constructed by the control variables. The flow of simulated annealing algorithm (SA) is presented in Figure 3 [40], and the system parameters and control parameters are illustrated in Tables 1 and 2, respectively.

The evolution curve of the SA is depicted in Figure 4. It can be seen that the objective function converges to a minimum value after a certain number of iterations. The final parameters are defined according to the optimal results and actual sample, which are 


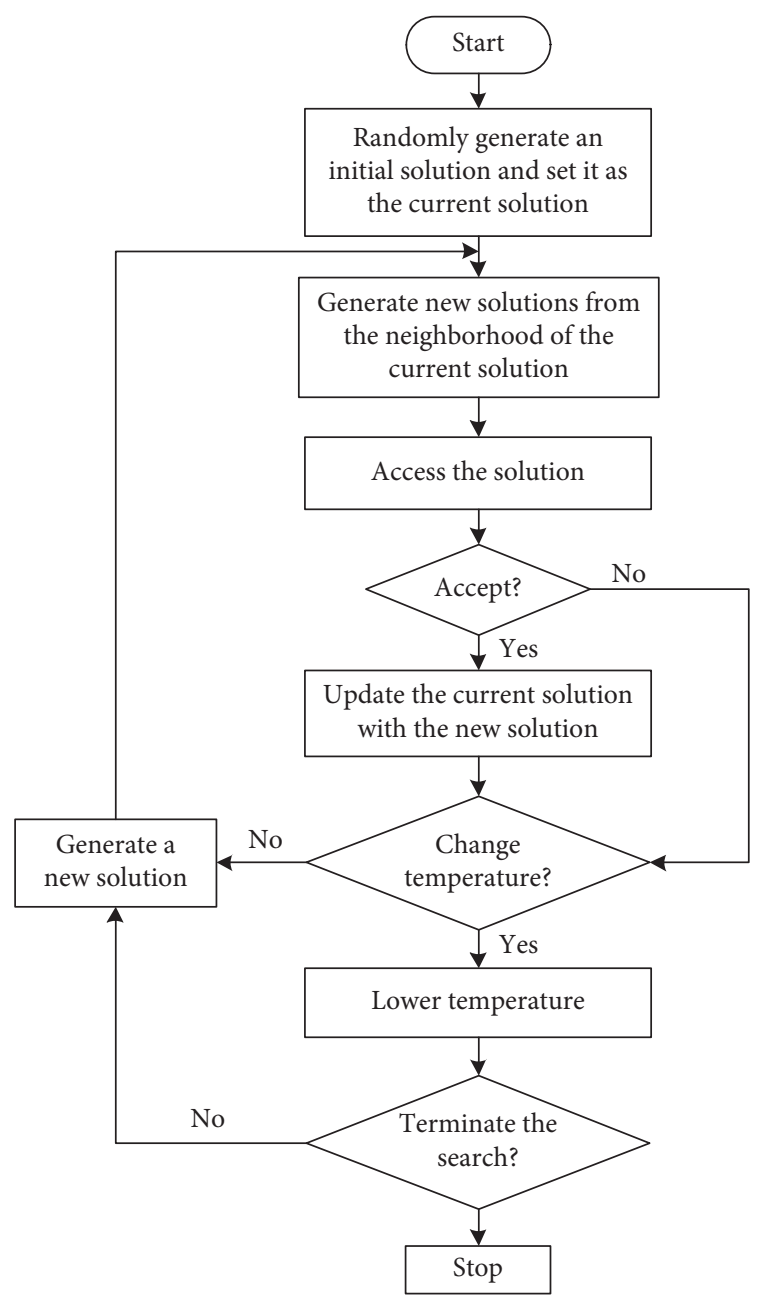

Figure 3: Flow of simulated annealing.

$$
\begin{aligned}
V_{0} & =6.3 \mathrm{~L}, \\
A_{a} & =1.0 \times 10^{-3} \mathrm{~m}^{2}, \\
p_{a 1} & =12 \mathrm{MPa}, \\
p_{a 2} & =13 \mathrm{MPa} .
\end{aligned}
$$

Remark 1. The desired trajectory may be different in diverse working conditions, which means the velocity in the lifting stage and retracted stage may change drastically. This configuration may not achieve the optimal effect in some extreme cases. However, it is most suitable for the situation that the trajectory in the retracted stage and lifting stage are symmetrical, which can be analyzed from Figure 2 .

\section{Principle of VSPC}

In the previous chapter, the optimized parameters are obtained so as to achieve better energy recuperation effect. However, the system efficiency can be further improved by regulating the supply pressure according to the demand.

\begin{tabular}{|c|c|c|c|}
\hline Variables & Description & Value & Unit \\
\hline$V_{0 \text { min }}$ & $\begin{array}{l}\text { Lower limit of the hydraulic } \\
\text { accumulator volume }\end{array}$ & 1 & $\mathrm{~L}$ \\
\hline$V_{0 \max }$ & $\begin{array}{l}\text { Upper limit of the hydraulic } \\
\text { accumulator volume }\end{array}$ & 8 & $\mathrm{~L}$ \\
\hline$A_{a \min }$ & $\begin{array}{c}\text { Lower limit of the effective area of } \\
\text { storage chamber }\end{array}$ & $0.3 \times 10^{-3}$ & $\mathrm{~m}^{2}$ \\
\hline$A_{a \max }$ & $\begin{array}{c}\text { Upper limit of the effective area of } \\
\text { storage chamber }\end{array}$ & $1.96 \times 10^{-3}$ & $\mathrm{~m}^{2}$ \\
\hline$p_{0 \text { min }}$ & $\begin{array}{l}\text { Lower limit of the precharge } \\
\text { pressure }\end{array}$ & 2 & Ml \\
\hline$p_{0 \max }$ & $\begin{array}{l}\text { Upper limit of the precharge } \\
\text { pressure }\end{array}$ & 10 & $\mathrm{MPa}$ \\
\hline$\alpha$ & Proportion & 0. & - \\
\hline G & Gravity of the mass load & 13250 & $\mathrm{~N}$ \\
\hline$L$ & Piston stroke of the actuator & 0.3 & $\mathrm{~m}$ \\
\hline
\end{tabular}
Therefore, the variable supply pressure control circuit (VSPC) is introduced.
TABLE 1: System parameters utilized in the SA.

TABLE 2: Control parameters utilized in the SA.

\begin{tabular}{lcc}
\hline Variables & Description & Value \\
\hline$\Gamma$ & Length of Markov chain & 200 \\
$\mathrm{~K}$ & Attenuation parameter & 0.998 \\
$S$ & Factor of step length & 0.01 \\
$T$ & Initial temperature & 100 \\
$Y Z$ & Tolerance & $1 \times 10^{-8}$ \\
\hline
\end{tabular}

The pump pressure in the traditional hydraulic system is usually a constant value, whereas the load pressure is always changing. This pressure difference may result in inferior energy efficiency. In contrast, the load-sensing system can regulate the supply pressure so as to meet the load demand. Therefore, a constant pressure margin between pump pressure and inlet pressure can be constructed. Despite the fact that the traditional load-sensing system can greatly improve the system efficiency, defects such as the measurement noise, response lag, pressure fluctuation, and stability problem cannot be ignored. To address the aforementioned problem and facilitate the design procedure, the VSPC which is based on the desired signals is presented in this study.

In the forward motion $\left(\dot{x}_{d} \geq 0\right)$, the ideal valve orifice equation can be expressed as follows by neglecting the uncertainties such as leakage [39]:

$$
\begin{aligned}
& A_{1} \dot{x}_{d}=k_{q} k_{x} u \sqrt{\frac{2}{\rho} \Delta p_{1}}, \\
& A_{2} \dot{x}_{d}=k_{q} k_{x} u \sqrt{\frac{2}{\rho} \Delta p_{2}},
\end{aligned}
$$

where $x_{d}$ is the desired trajectory and $\Delta p_{1}$ and $\Delta p_{2}$ are pressure differences across the valve.

Since the discrepancy between supply pressure and inlet chamber pressure should be kept as a constant value to save energy in VSPC, the desired pressure difference can be given as 


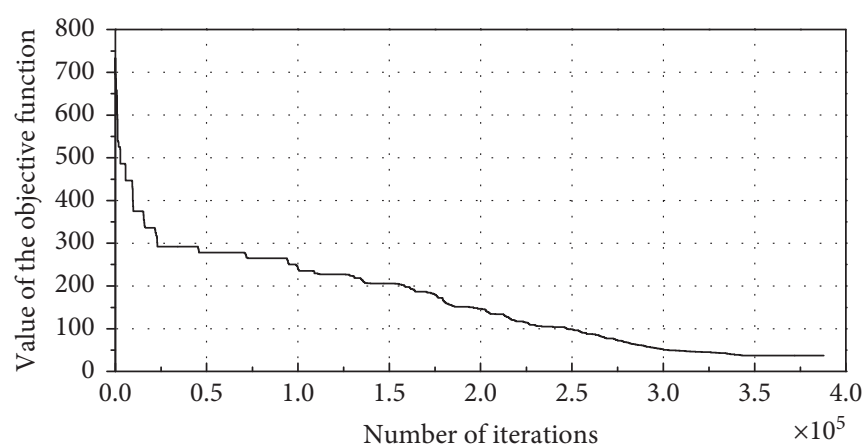

FIgURE 4: Evolution curve of the SA.

$$
\begin{aligned}
& \Delta p_{1}=p_{s}-p_{1}=p_{m}, \\
& \Delta p_{2}=p_{2}-p_{t}=p_{2},
\end{aligned}
$$

where $p_{m}$ is the constant pressure margin, which can be determined by the users/designers, and $p_{t}$ is assumed as zero.

Then, the desired input signal of PDV gives

$$
\bar{u}=\frac{A_{1} \dot{x}_{d}}{k_{q} k_{x}} \sqrt{\frac{\rho}{2 p_{m}}},
$$

where $\bar{u}$ is the desired input signal of PDV when the pressure margin is $p_{m}$.

Substituting equations (14) and (16) into equation (15), the desired outlet pressure can be derived as

$$
\bar{p}_{2}=\frac{\rho A_{2}^{2} \dot{x}_{d}^{2}}{2 k_{q}^{2} k_{x}^{2} \bar{u}^{2}}=\frac{p_{m} A_{2}^{2}}{A_{1}^{2}} .
$$

The desired pressure of the hydraulic accumulator is expressed as

$$
\bar{p}_{a}=p_{a 0}-\int \frac{p_{0} n}{V_{0}} A_{a} \dot{x}_{d} \mathrm{~d} t
$$

where $p_{a 0}$ is the initial pressure of the hydraulic accumulator.

The desired inlet pressure can be calculated by force balance equation [26]:

$$
\bar{p}_{1}=\frac{m}{A_{1}}\left(\ddot{x}_{d}+\frac{B_{c} \dot{x}_{d}}{m}+\frac{k x_{d}}{m}+\frac{G}{m}-\hat{\varphi}\right)+a_{1} \bar{p}_{2}-a_{2} \bar{p}_{a},
$$

where $\widehat{\varphi}_{1}$ is the estimate of $\varphi_{1}$, which will be synthesized later.

Therefore, the approximate inlet pressure and supply pressure can be given as

$$
p_{s}=\bar{p}_{1}+p_{m}
$$

Similar to the case of forward motion, the desired twochamber pressures and supply pressure when $\dot{x}_{d}<0$ are expressed as

$$
\begin{aligned}
& \bar{p}_{1}=\frac{p_{m} A_{1}^{2}}{A_{2}^{2}} \\
& \bar{p}_{2}=-\frac{m}{A_{2}}\left(\ddot{x}_{d}+\frac{B_{c} \dot{x}_{d}}{m}+\frac{k x_{d}}{m}+\frac{G}{m}-\widehat{\varphi}\right)+\frac{\bar{p}_{1}}{a_{1}}+\frac{a_{2} \bar{p}_{a}}{a_{1}} \\
& p_{s}=\bar{p}_{2}+p_{m} .
\end{aligned}
$$

Based on equations (19), (20), and (21), the VSPC can generate a control signal for the PRV so as to regulate the supply pressure according to the demand.

Remark 2. It can be seen from equations (19), (20), and (21) that the desired supply pressure mainly consists of the desired signals, which can be regarded as the feedforward part. Moreover, the unknown part exists in the force balance equation is compensated by $\widehat{\varphi}$.

\section{RBF Neural Network-Based Robust Controller Design}

The previous section mainly concentrates on the energy efficiency of the system. Nevertheless, the tracking performance is also an important aspect for the hydraulic servo system. Hence, the RBF neural network-based robust controller is constructed to ensure the prescribed tracking effect in this chapter, and the detailed control scheme is depicted in Figure 5.

It can be seen from equation (8) that there exists the unknown function $\varphi$, which may affect the trajectory tracking accuracy as well as the precision of the load pressure estimation in VSPC. The RBF neural network has good generalization ability and simple structure. Therefore, it can avoid unnecessary and lengthy calculation compared with the traditional BP neural network. It is proved by numerous literatures that RBF neural networks can approximate any nonlinear function in a compact set [41]. In order to obtain the estimate of the $\varphi$, the RBF neural network is utilized to approximate the real value. Subsequently, the estimation result of $\varphi$ is employed to compensate for the uncertainty both in VSPC and robust controller. 


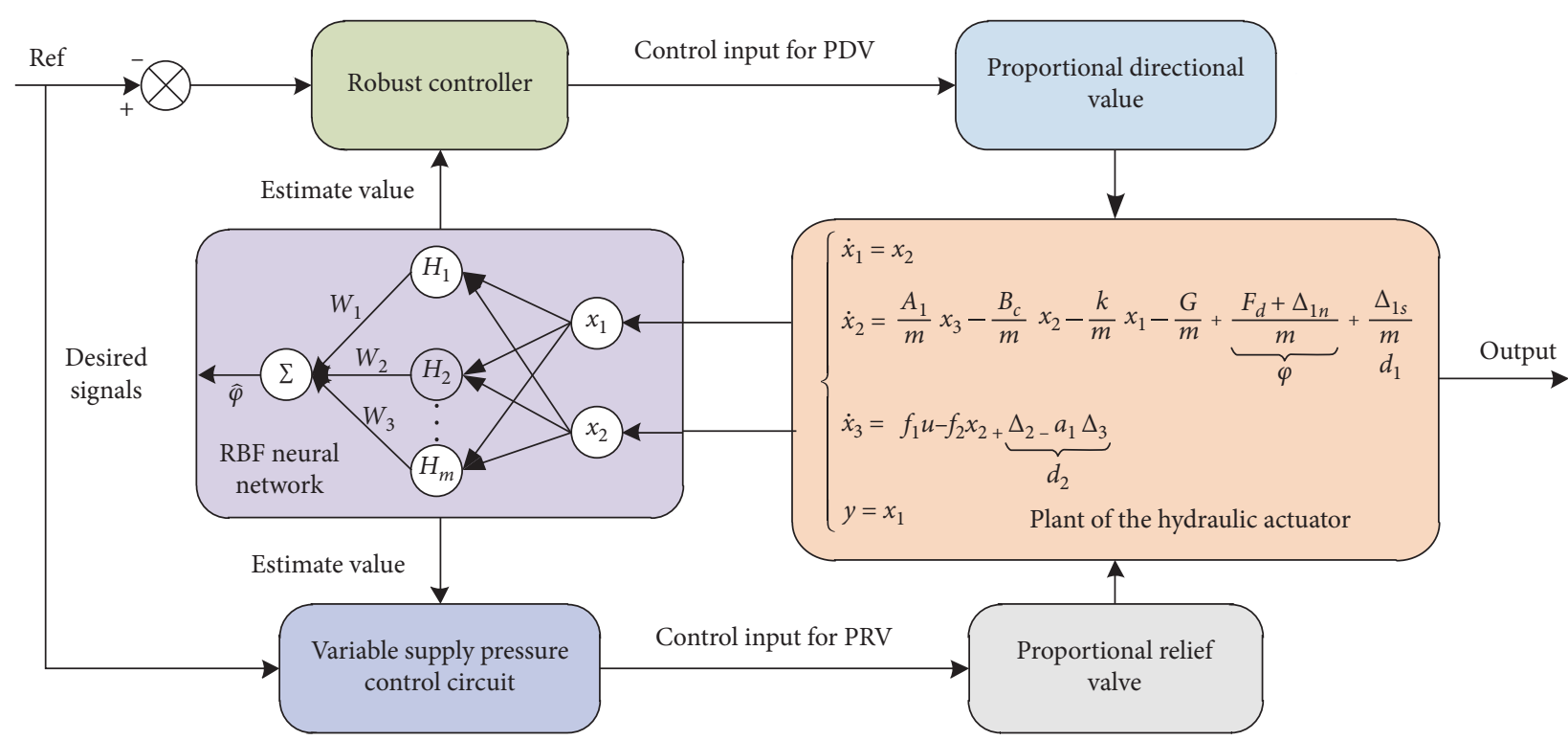

Figure 5: Detailed control scheme.

Step 1: define a switching-function-like quantity as [35]

$$
z_{2}=\dot{z}_{1}+k_{1} z_{1}=x_{2}-\alpha_{1}, \quad \alpha_{1}=\dot{x}_{d}-k_{1} z_{1},
$$

where $z_{1}=x_{1}-x_{d}$ denotes the tracking error and $k_{1}$ is a positive feedback gain.

Differentiating equation (22) and noting equation (8), one obtains

$$
\dot{z}_{2}=\frac{A_{1}}{m} x_{3}-\frac{B_{c}}{m} x_{2}-\frac{k}{m} x_{1}-\frac{G}{m}+\varphi+d_{1}-\ddot{x}_{d}+k_{1} x_{2}-k_{1} \dot{x}_{d} .
$$

The algorithm of the RBF neural network is expressed as

$$
\left\{\begin{array}{l}
H_{j}=\exp \left(\frac{\left\|\lambda-c_{j}\right\|^{2}}{2 b_{j}^{2}}\right), \\
\varphi=W^{* T} H(\lambda)+\varepsilon \\
\widehat{\varphi}=\widehat{W}^{T} H(\lambda), \\
\widetilde{\varphi}=\varphi-\widehat{\varphi}=\widetilde{W}^{T} H(\lambda)+\varepsilon
\end{array}\right.
$$

where $\lambda=\left[x_{1}, x_{2}\right]$ is the input of the RBF neural network, $W^{*}$ is the ideal weight, $H$ is the output of the Gaussian basis function, $j$ is the number of nodes in the hidden layer, $\varepsilon$ is the approximate error of the RBF neural network, and $\varepsilon \leq \varepsilon_{N}, \widehat{\varphi}$ is the estimate of $\varphi$ and $\widehat{W}$ is the estimate of $W^{*}, \widetilde{\varphi}$, and $\widetilde{W}$ are the estimation errors of the $\varphi$ and $W^{*}$, respectively.

Considering $x_{3}$ as the virtual control input, the control law $\alpha_{2}$ for $x_{3}$ is synthesized as follows [42]:

$$
\begin{aligned}
\alpha_{2} & =\alpha_{2 a}+\alpha_{2 s 1}+\alpha_{2 s 2}, \\
\alpha_{2 a} & =\frac{m}{A_{1}}\left(\frac{B_{c}}{m} x_{2}+\frac{k}{m} x_{1}+\frac{G}{m}-\widehat{\varphi}+\ddot{x}_{d}-k_{1} x_{2}+k_{1} \dot{x}_{d}-z_{1}\right), \\
\alpha_{2 s 1} & =-\frac{k_{2} m}{A_{1}} z_{2}, \\
\alpha_{2 s 2} & =-\frac{h_{2} m}{A_{1}} \tanh \left(\frac{z_{2}}{\eta_{2}}\right),
\end{aligned}
$$

where $\alpha_{2 a}$ is the model compensation term which functions as the feedforward part, the nominal system is stabilized by $\alpha_{2 s 1}, k_{2}$ is a positive feedback gain, the robust term $\alpha_{2 s 2}$ is utilized to suppress the lumped uncertainties, and $h_{2} \geq D_{1}+\varepsilon_{N}, \eta_{2}$ is the boundary-layer thickness.

Let $z_{3}=x_{3}-\alpha_{2}$ represent the input discrepancy, substituting equation (25) into equation (23) leads to

$$
\dot{z}_{2}=\frac{A_{1}}{m} z_{3}-z_{1}+\widetilde{\varphi}+d_{1}-k_{2} z_{2}-h_{2} \tanh \left(\frac{z_{2}}{\eta_{2}}\right),
$$

where $\widetilde{\varphi}=\varphi-\widehat{\varphi}$ denotes the estimation error of $\varphi$.

Step 2: in step 1, the robust virtual control law $\alpha_{2}$ has been developed. In this step, the actual control law $u$ has to be obtained. According to equation (8), the time derivative of $z_{3}$ can be expressed as

$$
\dot{z}_{3}=f_{1} u-f_{2} x_{2}+d_{2}-\dot{\alpha}_{2 c}-\dot{\alpha}_{2 u},
$$

where $\dot{\alpha}_{2 c}=\partial \alpha_{2} / \partial t+\left(\partial \alpha_{2} / \partial x_{1}\right) x_{2}+\left(\partial \alpha_{2} / \partial \widehat{\varphi}\right) \dot{\hat{\varphi}}$ denotes the calculable part of $\dot{\alpha}_{2}$ and $\dot{\alpha}_{2 u}=\partial \alpha_{2} / \partial x_{2}\left(\widetilde{\varphi}+d_{1}\right)$ represents the incalculable part of $\dot{\alpha}_{2}$. 
Therefore, the actual control law $u$ can be given as

$$
\begin{aligned}
u & =u_{a}+u_{s 1}+u_{s 2}, \\
u_{a} & =\frac{1}{f_{1}}\left(f_{2} x_{2}+\dot{\alpha}_{2 c}-\frac{A_{1}}{m} z_{2}\right), \\
u_{s 1} & =-\frac{k_{3}}{f_{1}} z_{3}, \\
u_{s 2} & =-\frac{h_{3}}{f_{1}} \tanh \left(\frac{z_{3}}{\eta_{3}}\right),
\end{aligned}
$$

where $u_{a}$ is the feedforward control law based on the model, the nominal system is stabilized by $u_{s 1}, k_{3}$ is a positive feedback gain, the robust term $u_{s 2}$ is utilized to suppress the lumped uncertainties, $\eta_{3}$ is the boundary-layer thickness, and $\quad h_{3} \geq\left|\partial \alpha_{2} / \partial x_{2}\right|\left(\left|\varphi_{M}\right|+D_{1}\right)+D_{2}$, in which $\varphi_{\mathrm{M}}=\varphi_{\max }-\varphi_{\text {min }}$.

Substituting equation (28) into equation (27) leads to

$$
\dot{z}_{3}=-\frac{A_{1}}{m} z_{2}-k_{3} z_{3}-h_{3} \tanh \left(\frac{z_{3}}{\eta_{3}}\right)+d_{2}-\dot{\alpha}_{2 u} .
$$

6.1. Main Results. The Lyapunov function is defined as [43]

$$
V=\frac{1}{2} z_{1}^{2}+\frac{1}{2} z_{2}^{2}+\frac{1}{2} z_{3}^{2}+\frac{1}{2} \gamma \widetilde{W}^{T} \tilde{W}
$$

where $\gamma$ is an adjustable positive factor.

Differentiating $V$ and noting equations (22), (24), (26), and (28) yields

$$
\begin{aligned}
\dot{V}= & z_{1} \dot{z}_{1}+z_{2} \dot{z}_{2}+z_{3} \dot{z}_{3}+\gamma \tilde{W}^{T} \dot{\hat{W}} \\
= & z_{1}\left(z_{2}-k_{1} z_{1}\right)+z_{2}\left[\frac{A_{1}}{m} z_{3}-z_{1}+\tilde{W}^{T} H(\lambda)+\varepsilon+d_{1}-k_{2} z_{2}\right. \\
& \left.-h_{2} \tanh \left(\frac{z_{2}}{\eta_{2}}\right)\right] \\
& +z_{3}\left[-\frac{A_{1}}{m} z_{2}-k_{3} z_{3}-h_{3} \tanh \left(\frac{z_{3}}{\eta_{3}}\right)+d_{2}-\dot{\alpha}_{2 u}\right]+\gamma \tilde{W}^{T} \dot{\hat{W}} \\
= & -k_{1} z_{1}^{2}-k_{2} z_{2}^{2}-k_{3} z_{3}^{2}+z_{2}\left[\varepsilon+d_{1}-h_{2} \tanh \left(\frac{z_{2}}{\eta_{2}}\right)\right] \\
& +z_{3}\left[d_{2}-\dot{\alpha}_{2 u}-h_{3} \tanh \left(\frac{z_{3}}{\eta_{3}}\right)\right]+\tilde{W}^{T}\left[z_{2} H(\lambda)+\gamma \dot{\hat{W}}\right] .
\end{aligned}
$$

The adaptive law of $W$ can be designed as

$$
\dot{\hat{W}}=-\frac{1}{\gamma} z_{2} H(\lambda)
$$

Substituting equation (31) into equation (30) gives

$$
\begin{aligned}
\dot{V}= & -k_{1} z_{1}^{2}-k_{2} z_{2}^{2}-k_{3} z_{3}^{2}+z_{2}\left[\varepsilon+d_{1}-h_{2} \tanh \left(\frac{z_{2}}{\eta_{2}}\right)\right] \\
& +z_{3}\left[d_{2}-\dot{\alpha}_{2 u}-h_{3} \tanh \left(\frac{z_{3}}{\eta_{3}}\right)\right] \leq 0 .
\end{aligned}
$$

Therefore, according to the lasalle unvarying theorem, the closed loop system is asymptotically stable.

\section{Analysis and Discussion of the Results}

In order to verify the aforementioned control strategy as well as the energy-saving effect, the entire model of the system is established. The model of the system is depicted in Figure 6, and the parameters are illustrated in Table 3. It should be noted that the three-chamber actuator is divided into two cylinders so as to facilitate the modelling process.

In order to verify the effectiveness of the proposed controller, a sinusoidal signal is utilized as the desired signal, i.e., $x_{d}=0.06 \sin (\pi t)$. The tracking errors of the presented controller and the PI controller are shown in Figure 7. The tracking performance of the PI controller is demonstrated for verification, and the parameters used in the PI controller are set to the values which can get the best results. It can be seen that the average tracking error of the proposed controller is lower than that of the PI controller, and the maximum tracking error is $2 \mathrm{~mm}$. Therefore, the tracking performance can meet the requirements in most cases.

The estimation value of unknown function $\varphi$ is depicted in Figure 8. It is obtained by using the RBF neural network. It is utilized to compensate for the uncertainty both in the VSPC and the robust controller. On account of the compensation function, the pump can exactly provide the demand pressure, which can be observed from Figures 9, 10, and 11.

The three controllers which were used for comparison are as follows.

System 1: the proposed system which comprises of the three-chamber cylinder and hydraulic accumulator is abbreviated as system 1 . The supply pressure is regulated according to the VSPC and the tracking performance is guaranteed by the RBF neural network-based robust controller.

System 2: in system 2, the VSPC is cancelled on the basis of system 1, and the other parts are retained. Therefore, the energy-saving effect of the VSPC can be observed intuitively by comparing system 1 and system 2. Moreover, the performance of energy recuperation can be analyzed by comparing system 2 and system 3 . System 3: the traditional hydraulic system is regarded as system 3. It usually comprises of a quantitative pump with constant supply pressure and a two chamber actuator (as shown in the right side of Figure 1). Hence, the comprehensive effect of energy recuperation and the VSPC can be obtained by comparing system 1 and system 3 . 


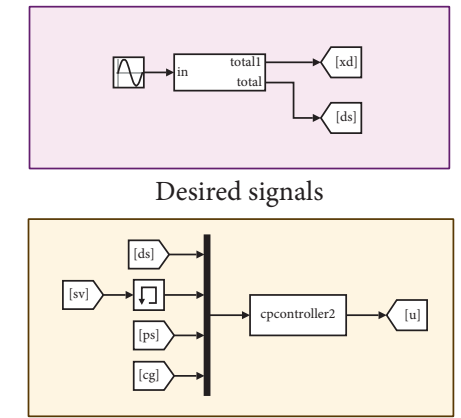

Robust controller and RBF neural network

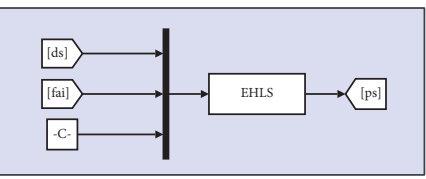

Electrohydraulic load-sensing

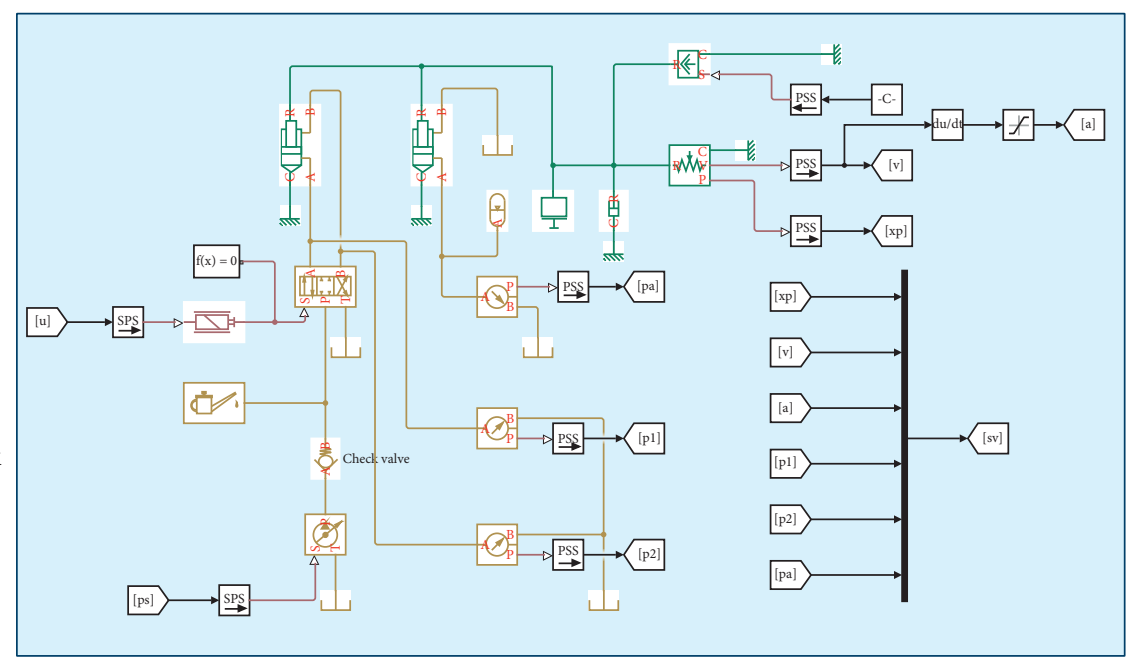

Model of the hydraulic actuator

Figure 6: Model of the entire system.

TABle 3: Parameters of the system.

\begin{tabular}{|c|c|c|c|}
\hline Variables & Description & Value & Unit \\
\hline$A_{1}$ & Effective area of the piston chamber & $0.962 \times 10^{-3}$ & $\mathrm{~m}^{2}$ \\
\hline$A_{2}$ & Effective area of the rod chamber & $0.945 \times 10^{-3}$ & $\mathrm{~m}^{2}$ \\
\hline$A_{a}$ & Effective area of the energy storage chamber & $1 \times 10^{-3}$ & $\mathrm{~m}^{2}$ \\
\hline$L$ & Piston stroke of the actuator & 0.3 & $\mathrm{~m}$ \\
\hline$B_{c}$ & Coefficient of viscous damping & 800 & $\mathrm{~N} / \mathrm{m} / \mathrm{s}$ \\
\hline$k$ & Stiffness of the environment & 0 & $\mathrm{~N} / \mathrm{m}$ \\
\hline G & Gravity of the mass load & 13250 & $\mathrm{~N}$ \\
\hline$\beta_{e}$ & Elasticity modulus of the oil & $1.46 \times 10^{3}$ & $\mathrm{MPa}$ \\
\hline$\rho$ & Density of the oil & 977 & $\mathrm{~kg} / \mathrm{m}^{3}$ \\
\hline$V_{0}$ & Total volume of the hydraulic accumulator & 6.3 & $\mathrm{~L}$ \\
\hline$n$ & Gas exponent & 1.4 & - \\
\hline$p_{a 1}$ & Minimum working pressure of the hydraulic accumulator & 12 & $\mathrm{MPa}$ \\
\hline$p_{a 2}$ & Maximum working pressure of the hydraulic accumulator & 13 & $\mathrm{MPa}$ \\
\hline$p_{0}$ & Precharge pressure of the hydraulic accumulator & 9.7 & $\mathrm{MPa}$ \\
\hline$p_{m}$ & Pressure margin of the VSPC & 0.5 & $\mathrm{MPa}$ \\
\hline$k_{1}$ & Control gain 1 & 400 & - \\
\hline$k_{2}$ & Control gain 2 & 400 & - \\
\hline$k_{3}$ & Control gain 3 & 400 & - \\
\hline$h_{1}$ & Control gain 4 & 200 & - \\
\hline$h_{2}$ & Control gain 5 & 200 & - \\
\hline
\end{tabular}

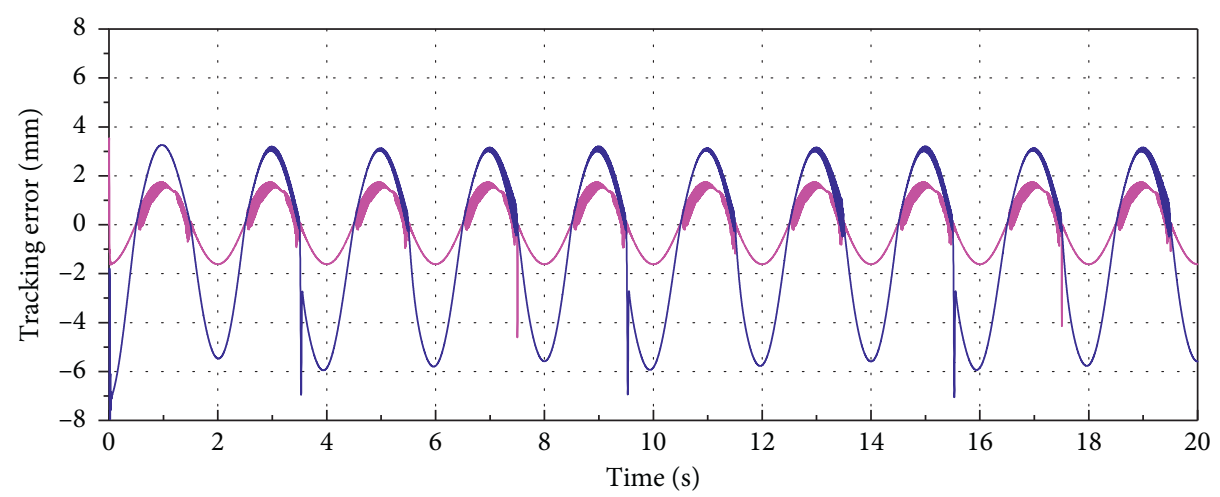

Tracking error of the presented controller Tracking error of the PID controller

Figure 7: Tracking error in test 1. 


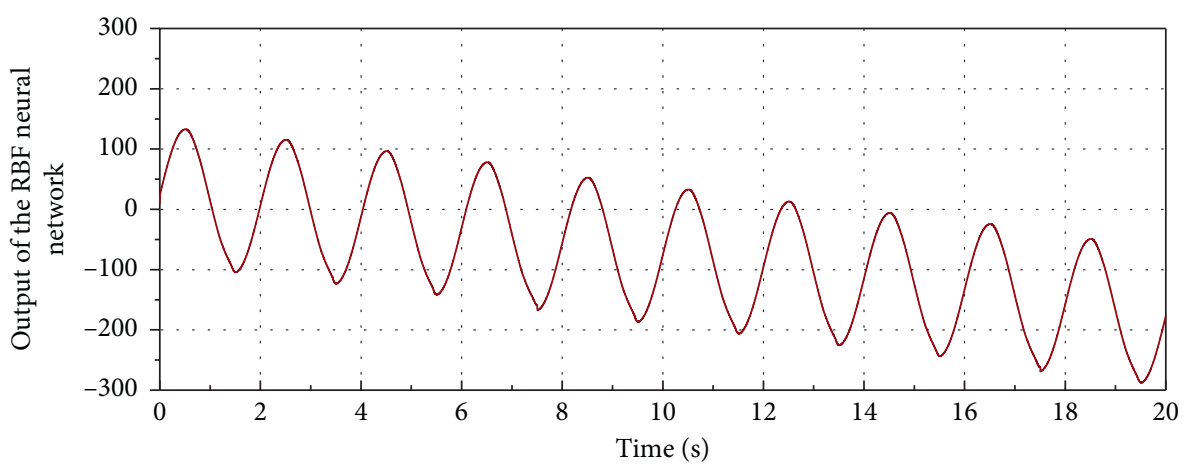

FIGURE 8: Output of the RBF neural network.

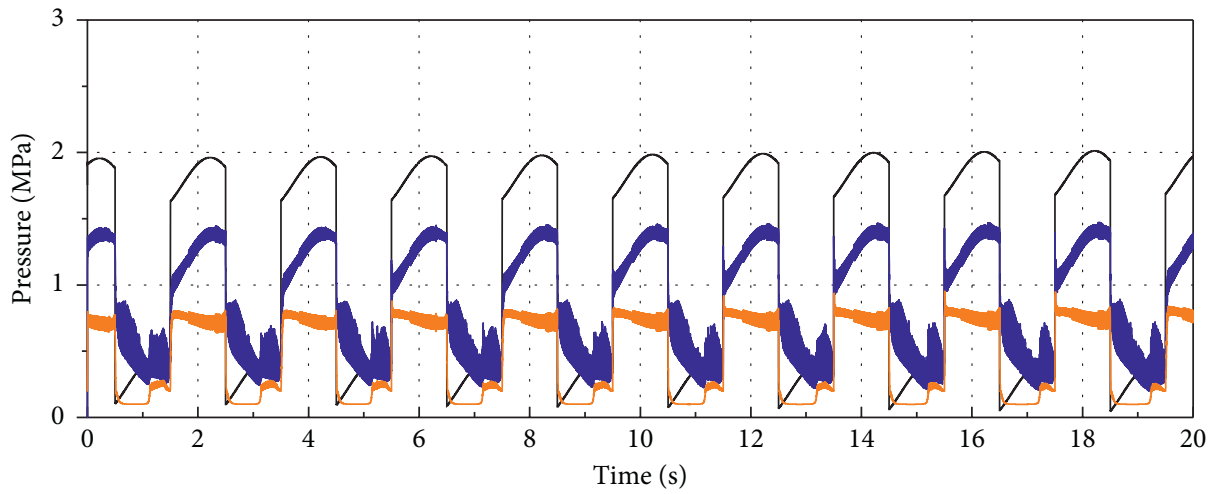

- Pressure of the pump

Pressure of the piston chamber

_ Pressure of the rod chamber

Figure 9: Pressures of system 1.

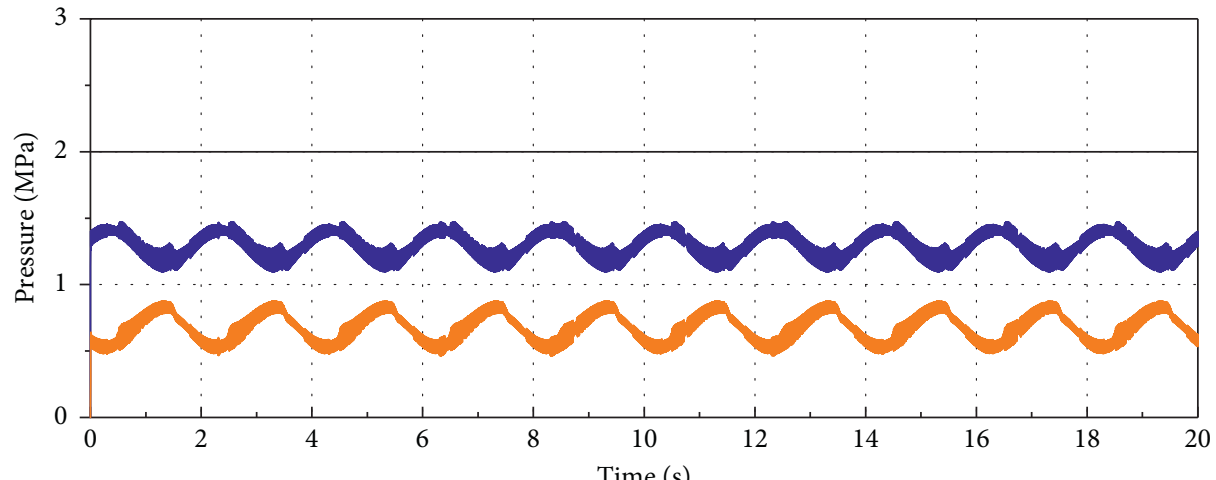

- Pressure of the pump

- Pressure of the piston chamber

_ Pressure of the rod chamber

Figure 10: Pressures of system 2.

The pressures of the three-chamber actuator are exhibited in Figures 9 and 12. It can be seen that a relatively low-supply pressure is required to drive the actuator. It is because the gravity of the mass load is balanced by the force provided by the hydraulic accumulator. Therefore, the demand energy is small either in the lifting stage or in the retracted stage. It should be noted that the pressure of the hydraulic accumulator does not vary between the maximum and minimum working pressures since the actuator moves in partial stroke. In addition, a notable feature of the presented system is that the pressure margin between the supply pressure and load pressure remains constant via VSPC, thereby saving energy. 


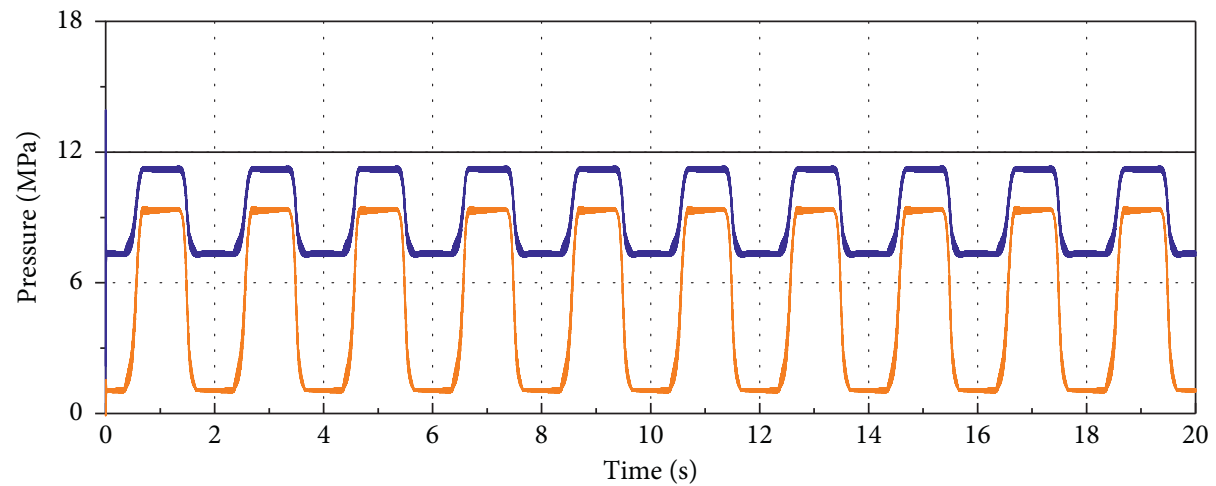

— Pressure of the pump

_ Pressure of the piston chamber

_ Pressure of the rod chamber

Figure 11: Pressures of system 3.

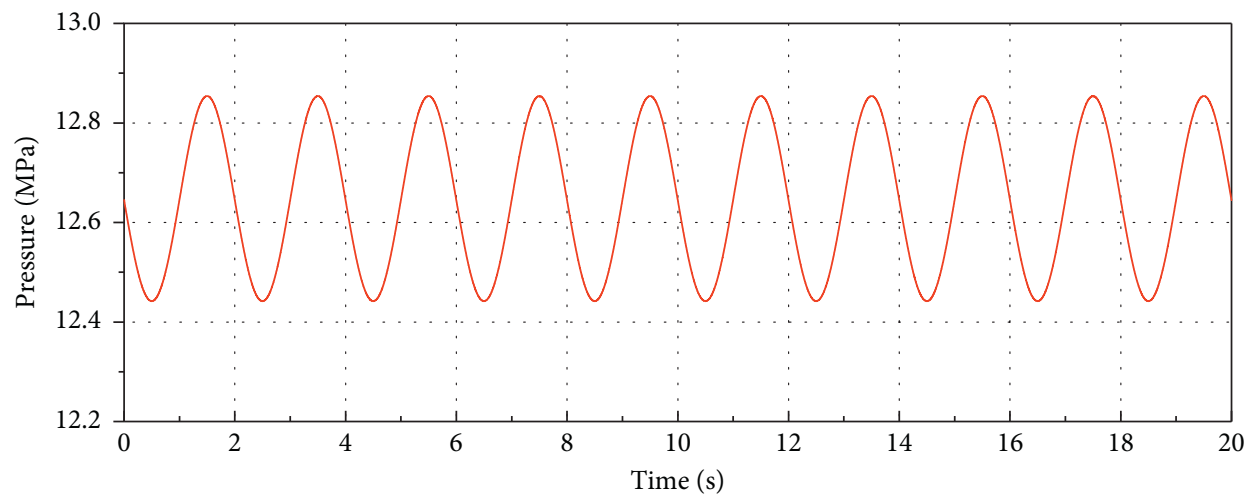

Figure 12: Pressure of the storage chamber.

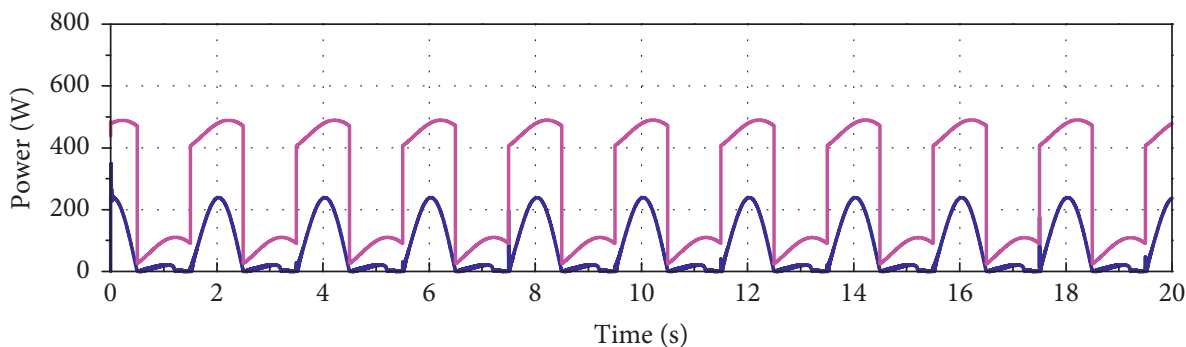

Effective power of system 1

Total power of system 1

(a)

Figure 13: Continued. 


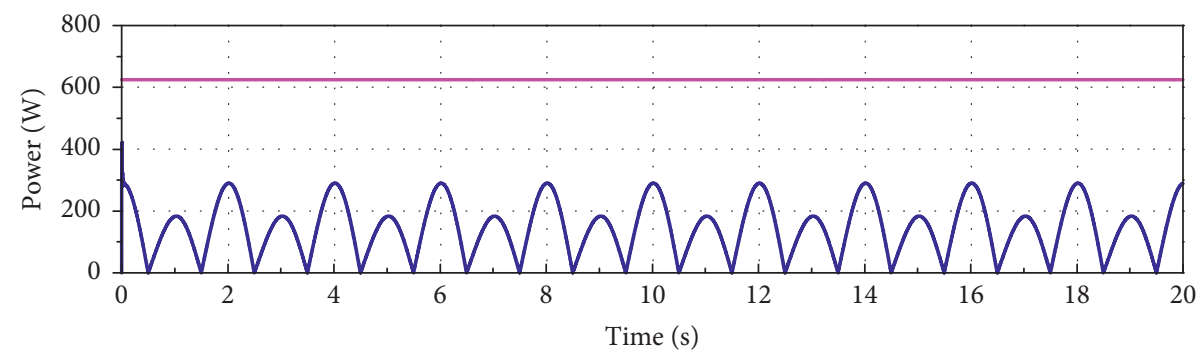

Effective power of system 2

Total power of system 2

(b)

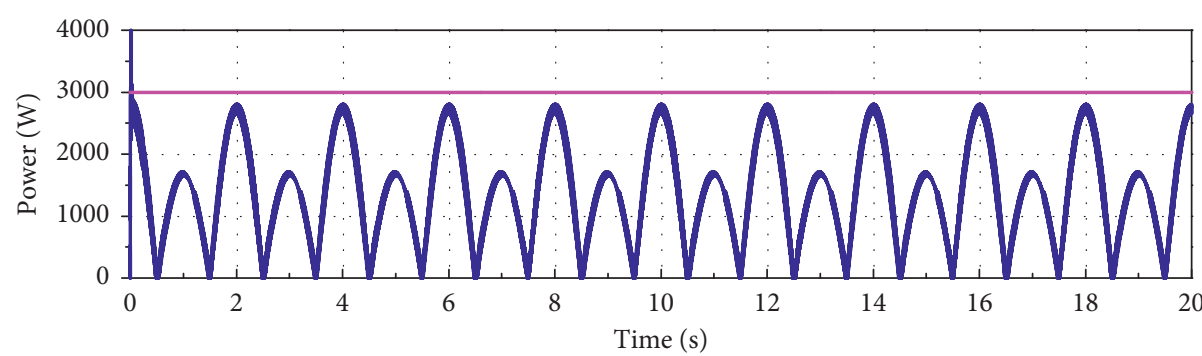

Effective power of system 3

Total power of system 3

(c)

FIgURE 13: The power of the three systems in test 1.

TABle 4: Parameter configurations for the hydraulic accumulator.

\begin{tabular}{|c|c|c|c|c|c|c|}
\hline \multirow{2}{*}{ Scheme } & \multicolumn{3}{|c|}{ Control variables } & \multicolumn{3}{|c|}{ Servo variables } \\
\hline & $p_{0}$ & $V_{0}$ & $A_{a}$ & $p_{a 1}$ & $p_{a 2}$ & $A_{1}$ \\
\hline Original & $9.7 \mathrm{MPa}$ & $6.3 \mathrm{~L}$ & $1.0 \times 10^{-3} \mathrm{~m}^{2}$ & $12 \mathrm{MPa}$ & $13 \mathrm{MPa}$ & $0.962 \times 10^{-3} \mathrm{~m}^{2}$ \\
\hline Set 1 & $8.7 \mathrm{MPa}$ & Invariable & Invariable & $11 \mathrm{MPa}$ & $12 \mathrm{MPa}$ & Invariable \\
\hline Set 2 & Invariable & $4 \mathrm{~L}$ & Invariable & Invariable & $13.6 \mathrm{MPa}$ & Invariable \\
\hline Set 3 & Invariable & Invariable & $0.5 \times 10^{-3} \mathrm{~m}^{2}$ & Invariable & $12.5 \mathrm{MPa}$ & $1.462 \times 10^{-3} \mathrm{~m}^{2}$ \\
\hline Set 4 & Invariable & Invariable & $1.5 \times 10^{-3} \mathrm{~m}^{2}$ & Invariable & $13.5 \mathrm{MPa}$ & $0.462 \times 10^{-3} \mathrm{~m}^{2}$ \\
\hline
\end{tabular}

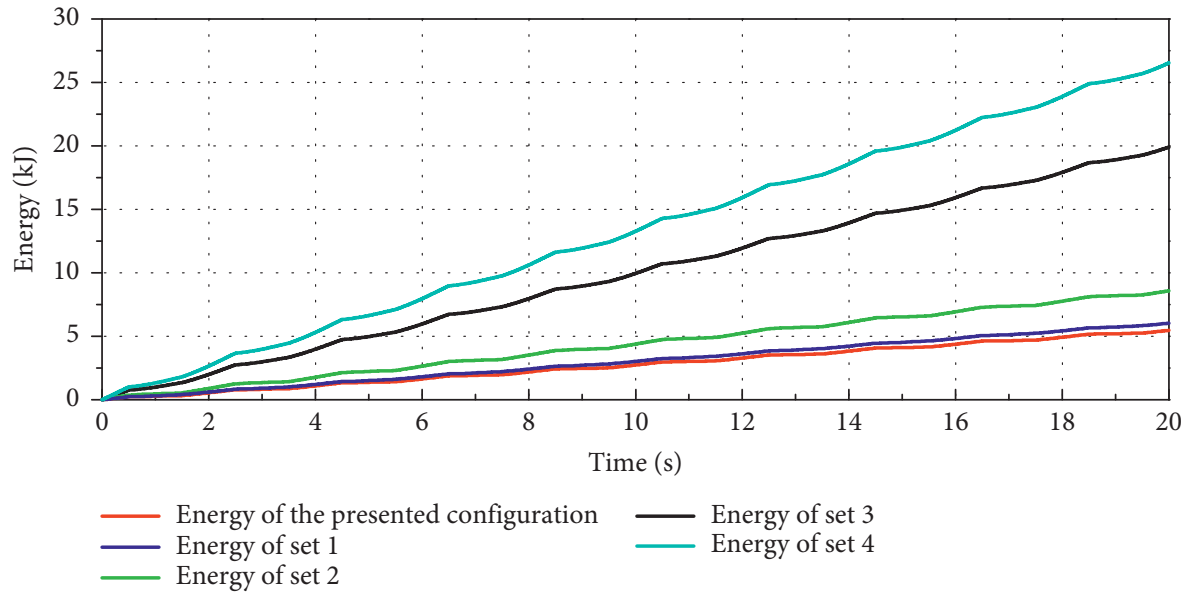

FIGURE 14: Comparison of the energy-saving effect of different parameter configurations of the hydraulic accumulator. 


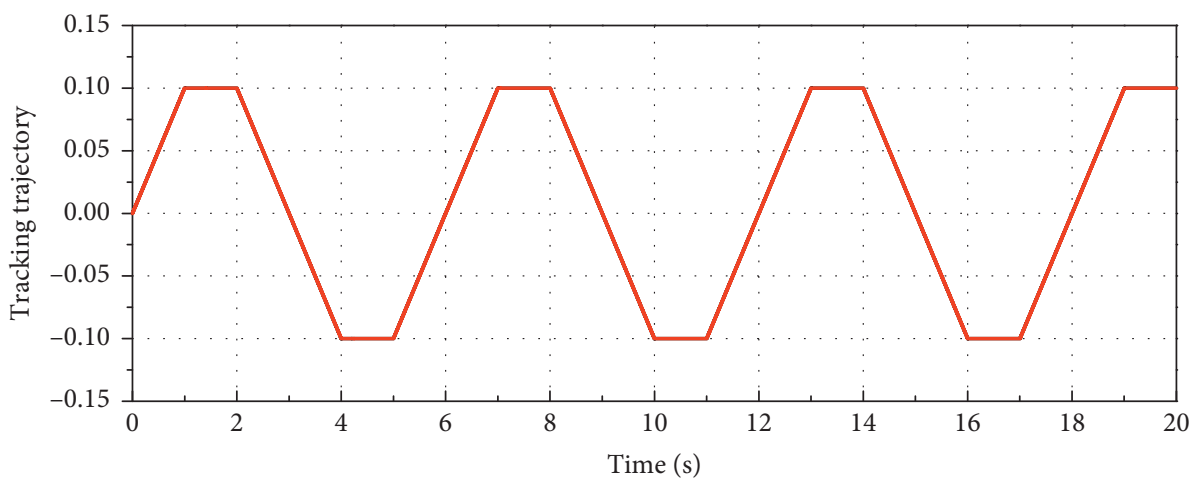

FIGURE 15: Desired trajectory in test 2.

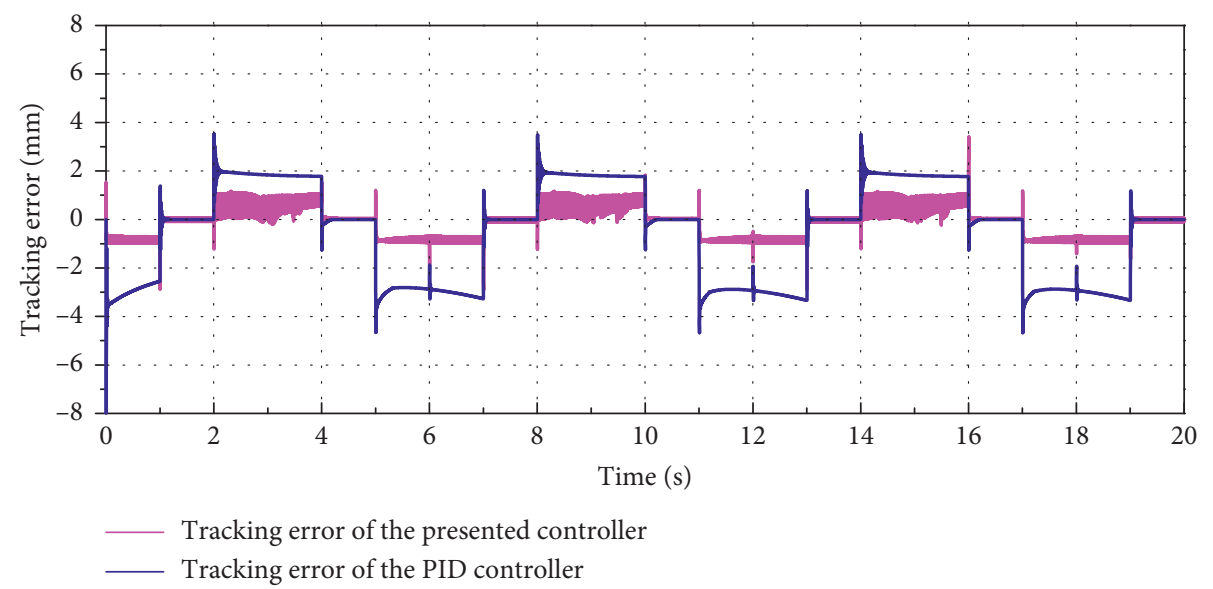

Figure 16: Tracking error in test 2.

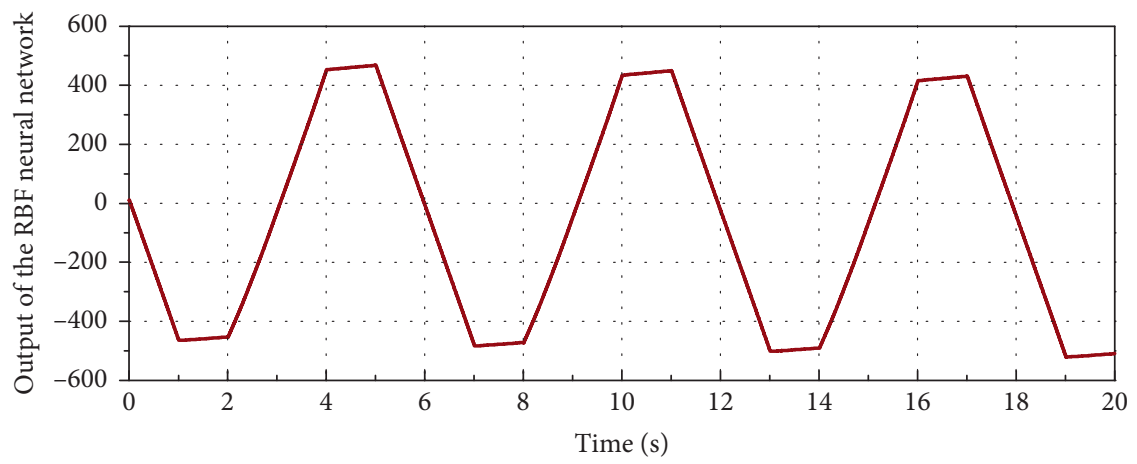

FIgURE 17: Output of the RBF neural network in test 2.

For the sake of contrast, the pressure of system 2 is depicted in Figure 10. Despite the fact that the maximum supply pressure in system 2 is identical with that in system 1 , there exists large excess pressure compared with system 1. Intuitively, system 3, which is shown in Figure 11, is energy inefficient since the pump should provide sufficient energy to actuate the mass load without the aid of the hydraulic accumulator. It should be noted that the constant supply pressure utilized in system 3 is the minimum pressure to meet the load requirements. The power of the system can be calculated by multiplying pressure and flow, and the power of the three system are shown in Figure 13. It can also be analyzed from Figure 13 that the peak power is about $500 \mathrm{~W}$ for the presented system, and it is one-sixth of the power in system 3 .

For the sake of verifying the effectiveness of the Simulated Annealing Algorithm, a variety of different parameter configurations are used for comparison (set 1 set 4), which is illustrated in Table 4. To analyze each variable on energy-saving effect, only one control variable is changed in each scheme. It can be seen from Figure 14 that the parameter configuration deduced by the SA is optimal, and the area of storage chamber has the largest impact on energy conservation. 


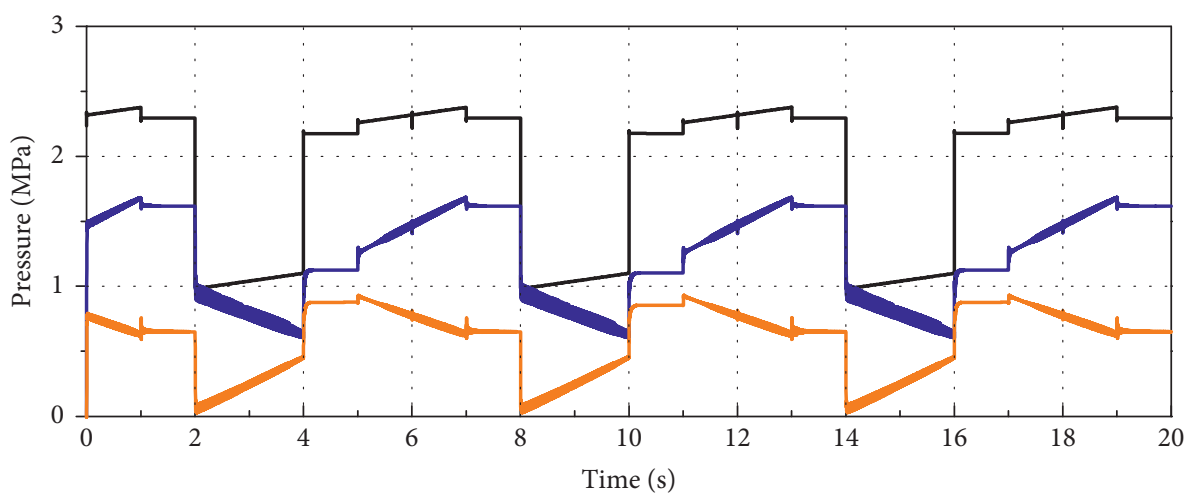

Pressure of the pump

Pressure of the piston chamber

Pressure of the rod chamber

Figure 18: Pressures of system 1 in test 2.

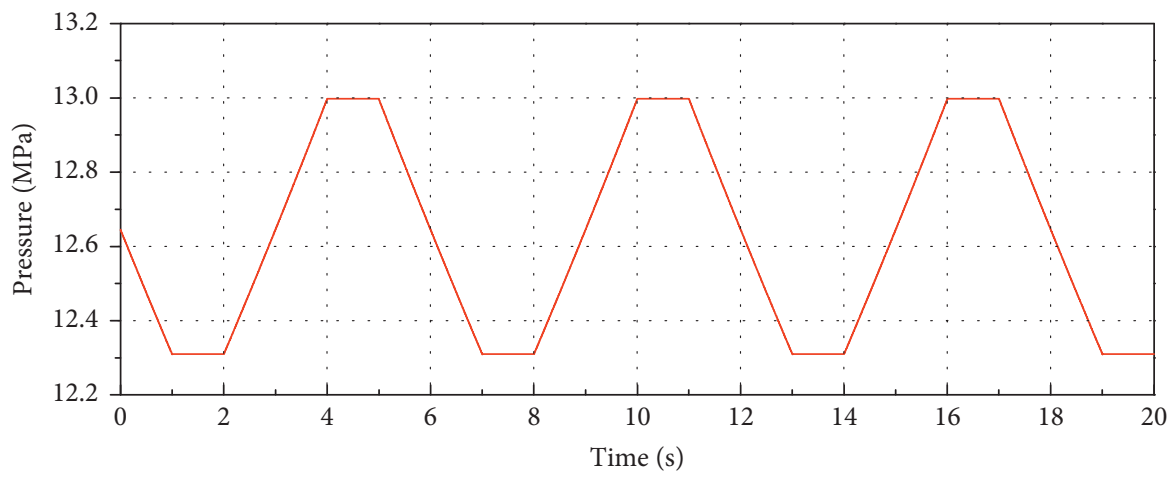

Figure 19: Pressure of the storage chamber in test 2.

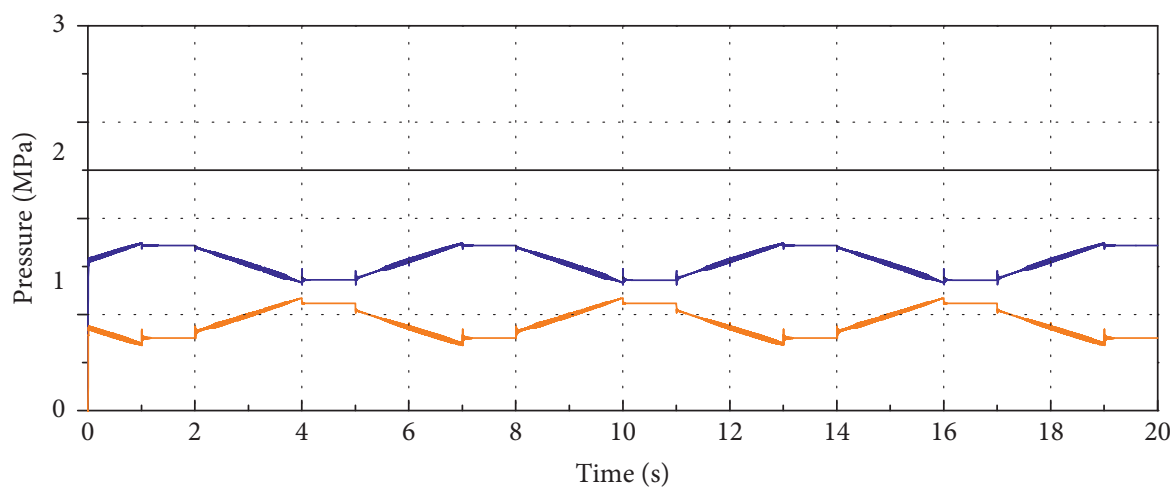

Pressure of the pump

Pressure of the piston chamber

Pressure of the rod chamber

Figure 20: Pressures of system 2 in test 2.

In order to further validate the effectiveness of the presented system, test 2 is conducted. The desired trajectory is shown in Figure 15.

The tracking errors of the presented controller and the PI controller are shown in Figure 16. It can be seen that the proposed controller can achieve satisfied tracking performance, and the maximum tracking error is about $2 \mathrm{~mm}$. The estimation of the unknown function is depicted in Figure 17. The pressures of the three-chamber actuator are demonstrated in Figures 18 and 19. Since there is an invariant phase in the desired trajectory, a straight line appears in the pressure curve of the storage chamber. The pressure 


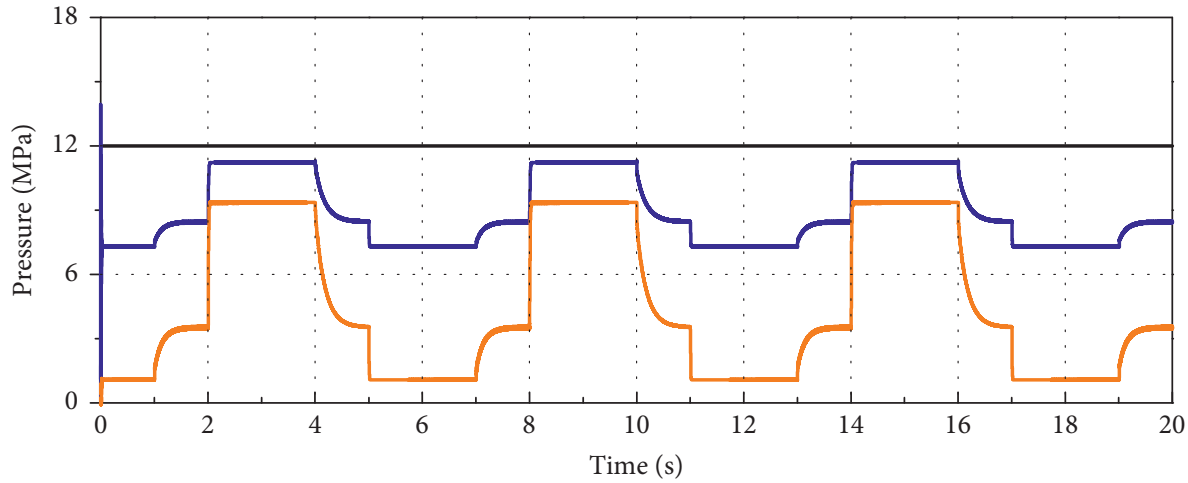

- Pressure of the pump

— Pressure of the piston chamber

_ Pressure of the rod chamber

Figure 21: Pressures of system 3 in test 2.

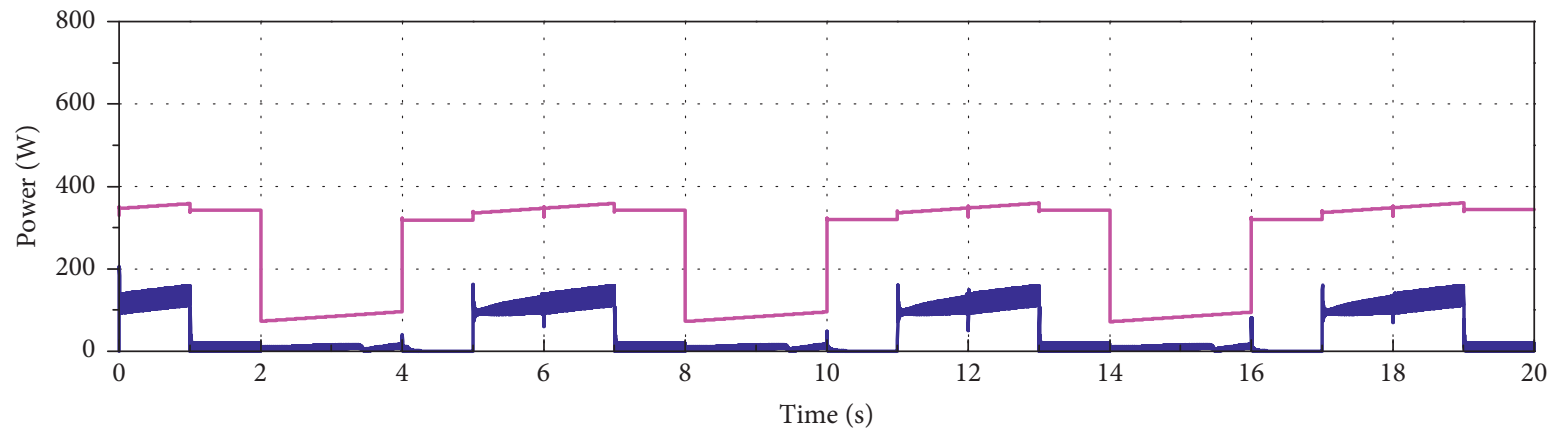

Effective power of system 1

Total power of system 1

(a)

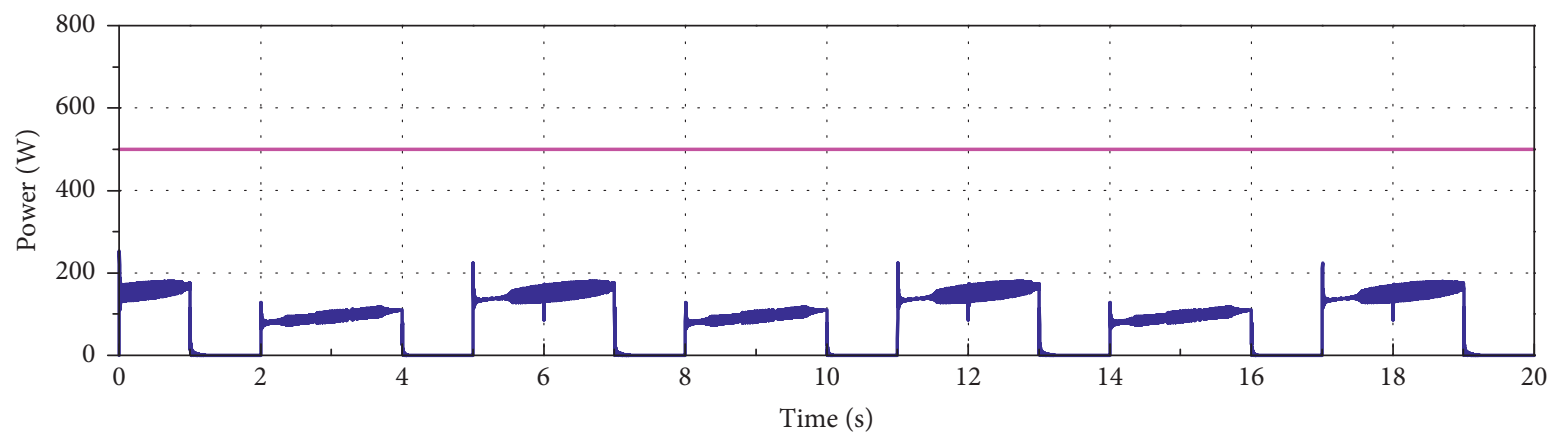

Effective power of system 2

Total power of system 2

(b)

Figure 22: Continued. 


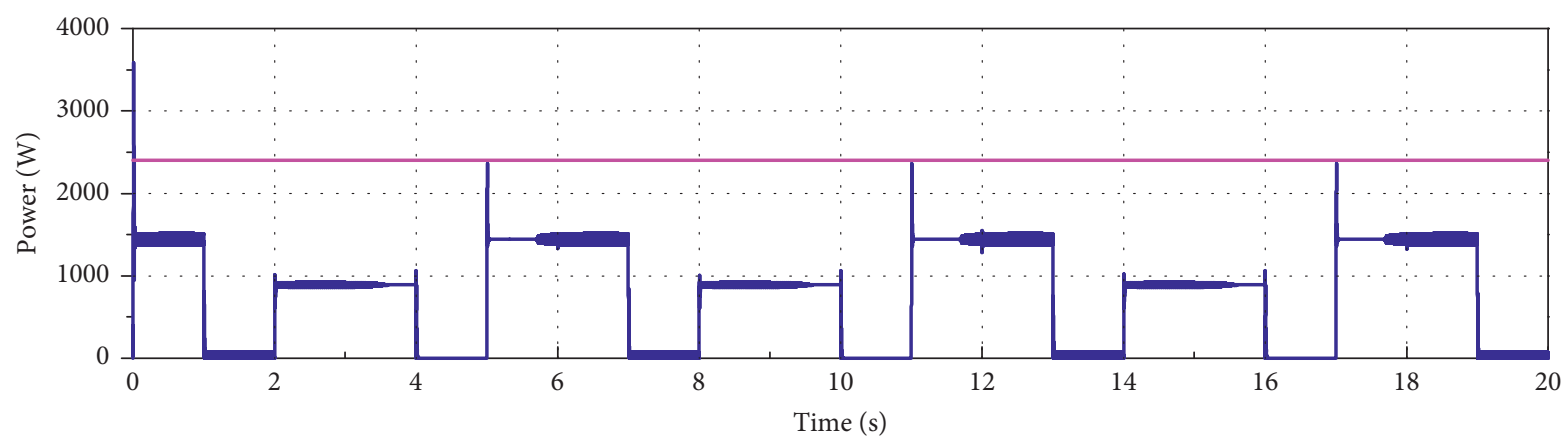

Effective power of system 3

Total power of system 3

(c)

FIgURE 22: The power of the three systems in test 2.

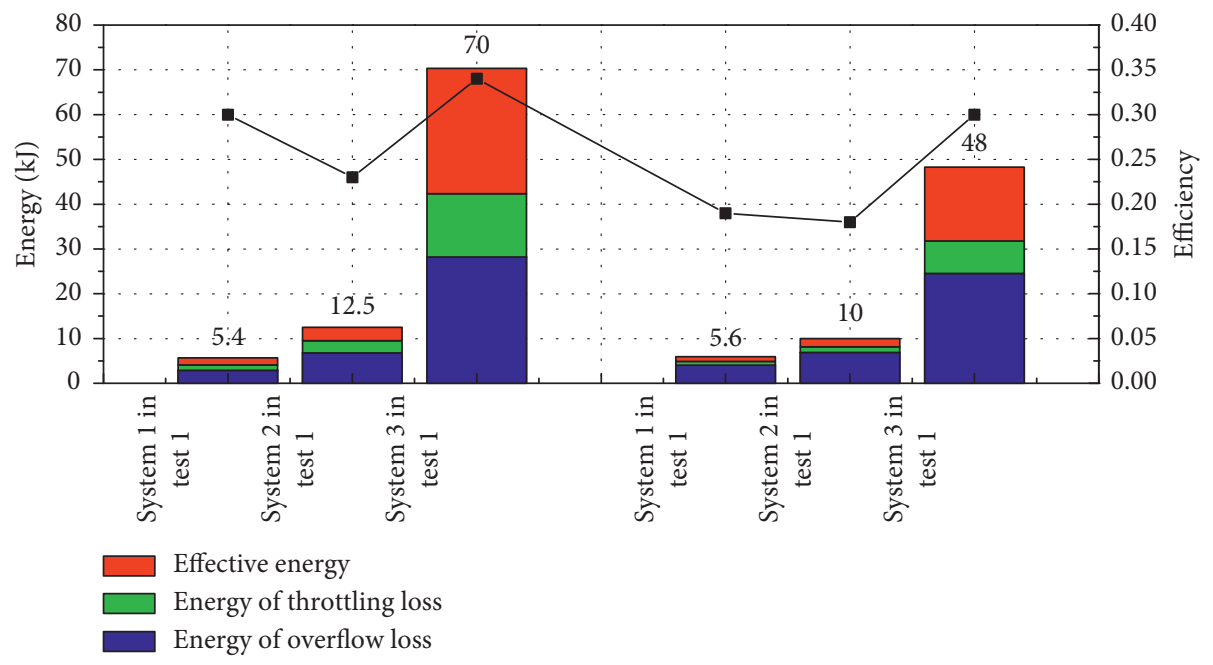

FIGURE 23: Energy distribution and contrast of the three systems.

curves of system 2 and system 3 are shown in Figures 20 and 21 , respectively. It can be observed from the pressure curves that the presented system is able to achieve significant energy-saving effect. The power of the three systems in test 2 is given in Figure 22, and the energy distribution in the two tests are depicted in Figure 23. Although the efficiency of system 3 is slightly higher than system 1, the energy consumption of system 1 is much lower than that of system 3 . Therefore, it can be concluded that the presented system can greatly reduce the energy dissipation.

\section{Conclusion}

To achieve the reduction of input energy, as well as precise trajectory tracking, an energy-efficient robust control for direct drive and energy recuperation hydraulic servo system is presented in this study. The main results are summarized as follows:

(1) The proposed system configuration is able to save nearly 90 percent of the energy input compared with the traditional hydraulic system. Although the actual effect may be lower than this value due to the energy loss in the actuator, pipe, and other components, the energy-saving ability is demonstrated.

(2) The asymptotic stability is ensured by the proposed controller. The tracking accuracy is guaranteed and the maximum tracking error is about $2 \mathrm{~mm}$ in both sets tests.

(3) For the sake of verifying the effect of simulated annealing algorithm, five different parameter configurations are utilized. The results demonstrate that the parameters generated by the simulated annealing algorithm are optimal.

(4) By using the VSPC method, the supply pressure of the pump can be adjusted according to the demand.

(5) It can be inferred from the pressure of the storage chamber that the gravitational potential energy can be recovered by the accumulator.

(6) The desired trajectory can be effectively tracked via the RBF neural network-based robust controller.

It is known that the load and the desired trajectory in different working conditions are completely different. 
Therefore, further research will focus on the energy consumption analysis under asymmetric desired trajectory and variable load. Furthermore, relevant experimental data will be supplemented in future work.

\section{Nomenclature}

$V_{0}: \quad$ Volume of the hydraulic accumulator

$p_{0}$ : $\quad$ Precharge pressure

$p_{a 1}: \quad$ Minimum working pressure

$p_{a 2}: \quad$ Maximum working pressure

$V_{a 1}: \quad$ Gas volume when pressure is $p_{a 1}$

$V_{a 2}: \quad$ Gas volume when pressure is $p_{a 2}$

$p_{a}: \quad \quad \quad$ Pressure of the hydraulic accumulator

$V_{a}: \quad$ Volume of the hydraulic accumulator

$Q_{a}: \quad$ Flow of the hydraulic accumulator

$x_{p}: \quad$ Displacement of the cylinder rod

$A_{a}: \quad \quad \quad$ Area of the storage chamber

$V_{w}: \quad$ Effective working volume

$Q_{1}$ : $\quad$ Flow rate of the forward chamber

$Q_{2}$ : $\quad$ Flow rate of the return chamber

$j: \quad \quad$ Number of nodes in the hidden layer

$\varepsilon: \quad \quad \quad \quad$ Approximate error of the RBF neural network

$\Delta p_{i}, i=1,2$ : Pressure difference across the valve

$\bar{p}_{i}, i=1,2: \quad$ Desired chamber pressure

$\lambda$ : $\quad$ Input of the RBF neural network

$H: \quad$ Output of the Gaussian basis function

$p_{a 0}$ : Initial pressure of the hydraulic accumulator

$z_{i}, i=1,2,3$ : Tracking errors

$\alpha_{i}, i=1,2: \quad$ Virtual control law

$\eta_{i}, i=2,3: \quad$ Boundary layer thickness.

$\Delta_{i}, i=1,2,3$ : Lumped uncertainties

$p_{0 \text { min }}: \quad$ Lower limit of accumulator volume

$A_{a \text { min }}$ : Lower limit of accumulator volume

$V_{0 \max }: \quad$ Upper limit of accumulator volume

$A_{a \max }: \quad$ Upper limit of storage chamber

$p_{0 \max }: \quad$ Upper limit of precharge pressure

$V_{0 \text { min }}$ : Lower limit of accumulator volume

$B_{c}: \quad$ Coefficient of viscous damping

$\bar{u}: \quad$ Desired input signal of PDV

$\widetilde{\varphi}: \quad$ Estimation error of the $\varphi$

$\widetilde{W}: \quad$ Estimation error of $W^{*}$

$d_{i}$ : $\quad$ Comprehensive uncertainties

$n: \quad$ Gas exponent

$\Gamma: \quad$ Length of the Markov chain

K: $\quad$ Attenuation parameter

$S: \quad$ Factor of step length

$T: \quad$ Initial temperature

YZ: $\quad$ Tolerance

$\alpha: \quad$ Proportional coefficient

$L: \quad$ Stroke of the actuator

$m: \quad$ Mass of the load

$p_{1}: \quad$ Pressure of the piston chamber

$p_{2}: \quad$ Pressure of the rod chamber

$A_{1}: \quad$ Area of the piston chamber

$A_{2}$ : $\quad$ Area of the rod chamber

$k: \quad$ Environment stiffness

$G: \quad$ Equivalent load gravity

$F_{d}: \quad$ External disturbance

$\begin{array}{ll}k_{q}: & \text { Flow gain coefficient } \\ k_{x}: & \text { Constant of the valve } \\ p_{s}: & \text { Supply pressure } \\ p_{t}: & \text { Tank pressure } \\ \rho: & \text { Oil density } \\ \varepsilon_{N}: & \text { Upper limit of } \varepsilon \\ D_{i}, i=1,2: & \text { Upper limit of } d_{i} \\ \widehat{\varphi}: & \text { Estimate of } \varphi \\ \widehat{W}: & \text { Estimate of } W^{*} \\ W^{*}: & \text { Ideal weight } \\ x_{d}: & \text { Desired trajectory } \\ p_{m}: & \text { Constant pressure margin } \\ u: & \text { Input signal of PDV } \\ \beta_{e}: & \text { Effective bulk modulus } \\ k_{1}: & \text { Control gain } 1 \\ k_{2}: & \text { Control gain } 2 \\ k_{3}: & \text { Control gain } 3 \\ h_{1}: & \text { Control gain } 4 \\ h_{2}: & \text { Control gain } 5 \\ \gamma: & \text { Adjustable factor } \\ \varphi: & \text { Unknown functions. }\end{array}$

\section{Data Availability}

The data used to support the findings of this study are included within the article.

\section{Conflicts of Interest}

The authors declare that there are no conflicts of interest regarding the publication of this article.

\section{Acknowledgments}

This work was supported by the Natural Science Foundation of the Jiangsu Higher Education Institutions (Grant no. 19KJB460002) and Natural Science Foundation of Jiangsu Normal University (Grant no. 18XLRX004).

\section{References}

[1] H. Huang, X. Zou, L. Li, X. Li, and Z. Liu, "Energy-saving design method for hydraulic press drive system with multi motor-pumps," International Journal of Precision Engineering and Manufacturing-Green Technology, vol. 6, no. 2, pp. 223234, 2019.

[2] L. Ge, L. Quan, Y. Li, X. Zhang, and J. Yang, "A novel hydraulic excavator boom driving system with high efficiency and potential energy regeneration capability," Energy Conversion and Management, vol. 166, pp. 308-317, 2018.

[3] L. Li, H. Huang, F. Zhao, and Z. Liu, "Operation scheduling of multi-hydraulic press system for energy consumption reduction," Journal of Cleaner Production, vol. 165, pp. 14071419, 2017.

[4] X. Yan, B. Chen, D. Zhang, C. Wu, and W. Luo, "An energysaving method to reduce the installed power of hydraulic press machines," Journal of Cleaner Production, vol. 233, pp. 538545, 2019.

[5] N. Vafamand and M. Rakhshan, "Dynamic model-based fuzzy controller for maximum power point tracking of photovoltaic systems: a linear matrix inequality approach," 
Journal of Dynamic Systems, Measurement, and Control, vol. 139, no. 5, 2017.

[6] S. Bahrebar, F. Blaabjerg, H. Wang et al., "A novel type-2 fuzzy logic for improved risk analysis of proton exchange membrane fuel cells in marine power systems application," Energies, vol. 11, no. 4, p. 721, 2018.

[7] N. Vafamand, M. M. Arefi, M. H. Khooban, T. Dragicevic, and F. Blaabjerg, "Nonlinear model predictive speed control of electric vehicles represented by linear parameter varying models with bias terms," IEEE Journal of Emerging and Selected Topics in Power Electronics, vol. 7, no. 3, pp. 2081-2089, 2019.

[8] M. H. Khooban, N. Vafamand, and J. Boudjadar, "Tracking control for hydrogen fuel cell systems in zero-emission ferry ships," Complexity, vol. 2019, Article ID 5358316, 9 pages, 2019.

[9] N. Vafamand, M. H. Khooban, T. Dragicevic, F. Blaabjerg, and J. Boudjadar, "Robust non-fragile fuzzy control of uncertain DC microgrids feeding constant power loads," IEEE Transactions on Power Electronics, vol. 34, no. 11, pp. 11300-11308, 2019.

[10] M.-H. Khooban, N. Vafamand, T. Dragičević, and F. Blaabjerg, "Polynomial fuzzy model-based approach for underactuated surface vessels," IET Control Theory \& Applications, vol. 12, pp. 914-921, 2018.

[11] M. H. Khooban, N. Vafamand, T. Niknam, T. Dragicevic, and F. Blaabjerg, "Model-predictive control based on TakagiSugeno fuzzy model for electrical vehicles delayed model," IET Electric Power Applications, vol. 11, no. 5, pp. 918-934, 2017.

[12] M. T. Nguyen, T. D. Dang, and K. K. Ahn, "Application of electro-hydraulic actuator system to control continuously variable transmission in wind energy converter," Energies, vol. 12, no. 13, p. 2499, 2019.

[13] W. Zhao, Z. Luan, and C. Wang, "Parametric optimization of novel electric-hydraulic hybrid steering system based on a shuffled particle swarm optimization algorithm," Journal of Cleaner Production, vol. 186, pp. 865-876, 2018.

[14] J. Gong, D. Zhang, Y. Guo et al., "Power control strategy and performance evaluation of a novel electro-hydraulic energysaving system," Applied Energy, vol. 233-234, pp. 724-734, 2019.

[15] G. Skorek, "Study of losses and energy efficiency of hydrostatic drives with hydraulic cylinder," Polish Maritime Research, vol. 25, no. 4, pp. 114-128, 2018.

[16] J.-h. Zhang, G. Liu, R. DIng, K. Zhang, M. Pan, and S. Liu, “3D printing for energy-saving: evidence from hydraulic manifolds design," Energies, vol. 12, no. 13, p. 2462, 2019.

[17] L. Schmidt, S. Ketelsen, M. H. Brask, and K. A. Mortensen, "A class of energy efficient self-contained electro-hydraulic drives with self-locking capability," Energies, vol. 12, no. 10, p. 1866, 2019.

[18] W. Wang and B. Wang, "An energy-saving control strategy with load sensing for electro-hydraulic servo systems," Strojniški Vestnik- Journal of Mechanical Engineering, vol. 62, no. 12, pp. 709-716, 2016.

[19] S. H. Cho and P. Noskievič, "Position tracking control with load-sensing for energy-saving valve-controlled cylinder system," Journal of Mechanical Science and Technology, vol. 26, no. 2, pp. 617-625, 2012.

[20] D. Lovrec, M. Kastrevc, and S. Ulaga, "Electro-hydraulic load sensing with a speed-controlled hydraulic supply system on forming-machines," The International Journal of Advanced
Manufacturing Technology, vol. 41, no. 11-12, pp. 1066-1075, 2009.

[21] H. Ding, J. Zhao, G. Cheng, S. Wright, and Y. Yao, "The influence of valve-pump weight ratios on the dynamic response of leaking valve-pump parallel control hydraulic systems," Applied Sciences, vol. 8, no. 7, p. 1201, 2018.

[22] A. Tivay, M. Zareinejad, S. M. Rezaei, and K. Baghestan, "A switched energy saving position controller for variablepressure electro-hydraulic servo systems," ISA Transactions, vol. 53, no. 4, pp. 1297-1306, 2014.

[23] K. Baghestan, S. M. Rezaei, H. A. Talebi, and M. Zareinejad, "An energy-saving nonlinear position control strategy for electro-hydraulic servo systems," ISA Transactions, vol. 59, pp. 268-279, 2015.

[24] C. Du, A. R. Plummer, and D. N. Johnston, "Performance analysis of a new energy-efficient variable supply pressure electro-hydraulic motion control method," Control Engineering Practice, vol. 60, pp. 87-98, 2017.

[25] W. Wang and B. Wang, "Disturbance observer-based nonlinear energy-saving control strategy for electro-hydraulic servo systems," Advances in Mechanical Engineering, vol. 9, no. 5, Article ID 168781401770584, 2017.

[26] W. Wang, J. Zhao, and H. Ding, "Output feedback nonlinear energy-saving position control of electro-hydraulic asymmetric actuator," Proceedings of the Institution of Mechanical Engineers, Part I: Journal of Systems and Control Engineering, vol. 232, no. 3, pp. 233-243, 2018.

[27] A. Saeedzadeh, A. Tivay, M. Zareinejad, S. M. Rezaei, A. Rahimi, and K. Baghestan, "Energy-efficient hydraulic actuator position tracking using hydraulic system operation modes," Proceedings of the Institution of Mechanical Engineers, Part E: Journal of Process Mechanical Engineering, vol. 232, no. 1, pp. 49-64, 2018.

[28] K. Yang, Y. Li, L. Zhou, and X. Rong, "Energy efficient foot trajectory of trot motion for hydraulic quadruped robot," Energies, vol. 12, no. 13, p. 2514, 2019.

[29] R. Galluzzi, Y. Xu, N. Amati, and A. Tonoli, "Optimized design and characterization of motor-pump unit for energyregenerative shock absorbers," Applied Energy, vol. 210, pp. 16-27, 2018.

[30] N. Mousavi, G. Kothapalli, D. Habibi, M. Khiadani, and C. K. Das, "An improved mathematical model for a pumped hydro storage system considering electrical, mechanical, and hydraulic losses," Applied Energy, vol. 247, pp. 228-236, 2019.

[31] Y.-X. Yu and K. K. Ahn, "Optimization of energy regeneration of hybrid hydraulic excavator boom system," Energy Conversion and Management, vol. 183, pp. 26-34, 2019.

[32] R. Ding, J. Zhang, B. Xu, M. Cheng, and M. Pan, "Energy efficiency improvement of heavy-load mobile hydraulic manipulator with electronically tunable operating modes," Energy Conversion and Management, vol. 188, pp. 447-461, 2019.

[33] J. Shi, L. Quan, X. Zhang, and X. Xiong, "Electro-hydraulic velocity and position control based on independent metering valve control in mobile construction equipment," Automation in Construction, vol. 94, pp. 73-84, 2018.

[34] L. Lyu, Z. Chen, and B. Yao, "Development of pump and valves combined hydraulic system for both high tracking precision and high energy efficiency," IEEE Transactions on Industrial Electronics, vol. 66, no. 9, pp. 7189-7198, 2019.

[35] L. Lu and B. Yao, "Energy-saving adaptive robust control of a hydraulic manipulator using five cartridge valves with an accumulator," IEEE Transactions on Industrial Electronics, vol. 61, no. 12, pp. 7046-7054, 2014. 
[36] L. Xia, L. Quan, L. Ge, and Y. Hao, "Energy efficiency analysis of integrated drive and energy recuperation system for hydraulic excavator boom," Energy Conversion and Management, vol. 156, pp. 680-687, 2018.

[37] T. Koitto, H. Kauranne, O. Calonius, T. Minav, and M. Pietola, "Experimental study on fast and energy-efficient direct driven hydraulic actuator unit," Energies, vol. 12, no. 8, p. 1538, 2019.

[38] C. Campbell and C. Campbell, "Hydropneumatic suspension," Automobile Suspensions, pp. 143-164, Springer, Boston, MA, USA, 1981.

[39] P. Chalupa and J. Novák, "Modeling and model predictive control of nonlinear hydraulic system," Advances in Intelligent Systems and Computing, Springer, Berlin, Germany, 2013.

[40] E. Aarts, J. Korst, and W. Michiels, "Simulated annealing," Search Methodologies, Springer, Boston, MA, USA, 2005.

[41] T. Van Nguyen and C. Ha, "RBF neural network adaptive sliding mode control of rotary stewart platform," Intelligent Computing Methodologies, Springer, Cham, Switzerland, 2018.

[42] R. Outbib and M. Zasadzinski, "Sliding mode control," Control Methods for Electrical Machines, pp. 169-206, Wiley, Hoboken, NJ, USA, 2010.

[43] R. Colgren, "Applications of robust control to nonlinear systems," American Institute of Aeronautics and Astronautics, Reston, Virginia, 2004. 